\title{
Anaerobic conditioning of E. Coli Cell Lysate for Enhanced In vitro Protein Synthesis
}

By Denis Tamiev ${ }^{1}$, Jared L. Dopp ${ }^{2}$, Nigel F. Reuel ${ }^{2}$

1. Biochemistry, biophysics and molecular biology department at lowa State University, Ames, lowa, 50011

2. Chemical and biological engineering department at lowa State University, Ames, lowa, 50011

Send correspondence to reuel@iastate.edu

\section{Contents}

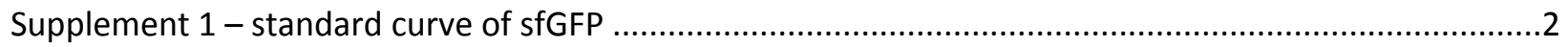

Supplement 2 - Comparing Aerobically and Anaerobically cultured Lysates............................................4

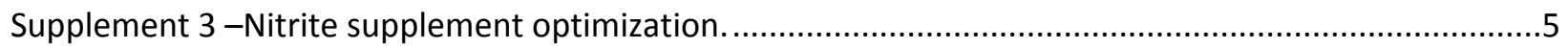

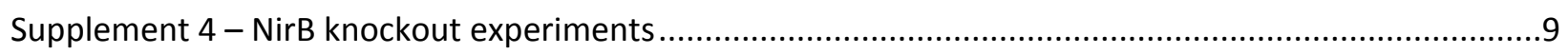

Supplement 5 - Anaerobic (glove box) CFPS reactions ....................................................................10

Supplement 6 - Relative loss or gain calculations and error propagation. ..........................................13

Supplement 7 - Luminescence experiments and ATP measurements...................................................14

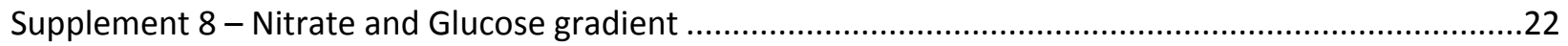

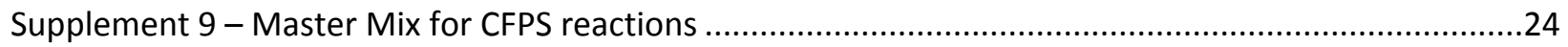

Supplement 10 - comparing various lysates to commercial kits.............................................................25

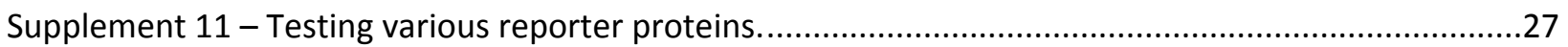




\section{Supplement 1 - standard curve of sfGFP}

Correlation of relative fluorescence units to physical units such as protein concentration in micrograms per milliliter can be achieved with a standard curve. In this work, we generated a standard curve by measuring protein concentration of a lysozyme solution using Pierce $660 \mathrm{~nm}$ protein concentration assay (Supplement Table 1). This method allowed us to generate a standard curve that can correlate absorbance at $660 \mathrm{~nm}$ and concentration of a protein solution with known concentration. The standard curve can then be used to correlate absorbance at $660 \mathrm{~nm}$ with unknown concentration of a protein solution, in this case, sfGFP (Supplement Figure 1A). In this experiment $10 \mu \mathrm{l}$ of the Lysozyme solution of known concentration was mixed with $150 \mu$ of the reagent in a 96 well plate. The reactions were incubated for $6 \mathrm{~min}$ at room temperature and the absorbance at $660 \mathrm{~nm}$ was measured. The experiment was performed in triplicates for each protein concentration. The effective range of the standard curve was determined to be from $1 \mathrm{mg} / \mathrm{ml}$ to $31.25 \mu \mathrm{g} / \mathrm{ml}$.

The pJL1 plasmid coding for the sfGFP reporter protein was transformed in BL21 DE3 cells and expressed in vivo. Protein expression was performed in LB media at 37C with IPTG induction. Cells were harvested and washed with S30 media via centrifugation and resuspension. Cells paste was resuspended in S30 media and $1 \mathrm{ml}$ aliquots were sonicated for 532 joules, at $50 \%$ amplitude and $10 \mathrm{on} / 10$ off intervals (identical to CFPS lysate sonication procedure). Sonicated cells were supplemented with PMSF protease inhibitor to prevent sfGFP degradation, and centrifuged at $4 \mathrm{C}$ for $10 \mathrm{~min}$ at $10,000 \mathrm{rcf}$. The supernatant was collected and combined (10ml of supernatant).

The supernatant was purified using the one step strep tag affinity chromatography purification procedure (Strep-Tactin II, Sigma Aldrich). The column ( $1 \mathrm{ml}$ bed volume) was washed with S30 buffer (10 column volumes) three times. Both, the column wash buffer and supernatant were supplemented with $5 \mathrm{mM}$ final concentration of 2-mercaptoethanol to minimize non-specific interactions with the affinity resin. Supernatant was applied to the column and washed with the column wash buffer (5 column volumes, 3 wash cycles). The protein of interest, sfGFP was eluted with $2.5 \mathrm{mM}$ desthibiotin. The entire purification procedure was performed in minimal light conditions to minimize photo degradation of sfGFP.

The fluorescence of the purified sfGFP was measured using the same conditions as throughout the project. A $13 \mu \mathrm{l}$ of purified sfGFP was applied to the 384 well plate, and the relative fluorescence was measured. Then, the concentration of the purified sfGFP was determined using the standard curve developed for lysozyme. That resulted in a standard curve that can be used to correlate relative fluorescence units (RFU) reported throughout the paper with protein concentration (Supplement Figure 1B). 
Supplement Table 1 - Concentration and absorbance of the standard Lysozyme solution, followed by concentration and corresponding relative fluorescence readings of sfGFP used to generate a standard curve (Supplement Figure 1B). Excitation at $488 \mathrm{~nm}$, emission at $509 \mathrm{~nm}$ with bandwidth of $10 \mathrm{~nm}$, and gain at 60.

\begin{tabular}{|c|c|c|c|c|c|c|}
\hline & \multicolumn{5}{|c|}{ Absorbance at $660 \mathrm{~nm}$} \\
\hline & & Trial 1 & Trial 2 & Trial 3 & AVG & STDEV \\
\hline \multirow{6}{*}{ 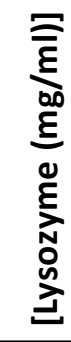 } & 1 & 0.571 & 0.559 & 0.549 & 0.560 & 0.011 \\
\hline & 0.5 & 0.355 & 0.369 & 0.371 & 0.365 & 0.009 \\
\hline & 0.25 & 0.253 & 0.253 & 0.251 & 0.252 & 0.001 \\
\hline & 0.125 & 0.195 & 0.201 & 0.202 & 0.199 & 0.004 \\
\hline & 0.0625 & 0.168 & 0.172 & 0.173 & 0.171 & 0.003 \\
\hline & 0.03125 & 0.154 & 0.161 & 0.16 & 0.158 & 0.004 \\
\hline \multirow{5}{*}{ 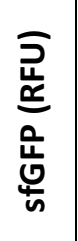 } & 8013.667 & 0.446 & 0.43 & 0.427 & 0.434 & 0.010 \\
\hline & 3912.333 & 0.294 & 0.291 & 0.292 & 0.292 & 0.002 \\
\hline & 1551.333 & 0.227 & 0.224 & 0.223 & 0.225 & 0.002 \\
\hline & 1125 & 0.186 & 0.189 & 0.183 & 0.186 & 0.003 \\
\hline & 547.6667 & 0.175 & 0.168 & 0.161 & 0.168 & 0.007 \\
\hline
\end{tabular}


A. Lysozyme Standard Curve Pierce 660nm Assay

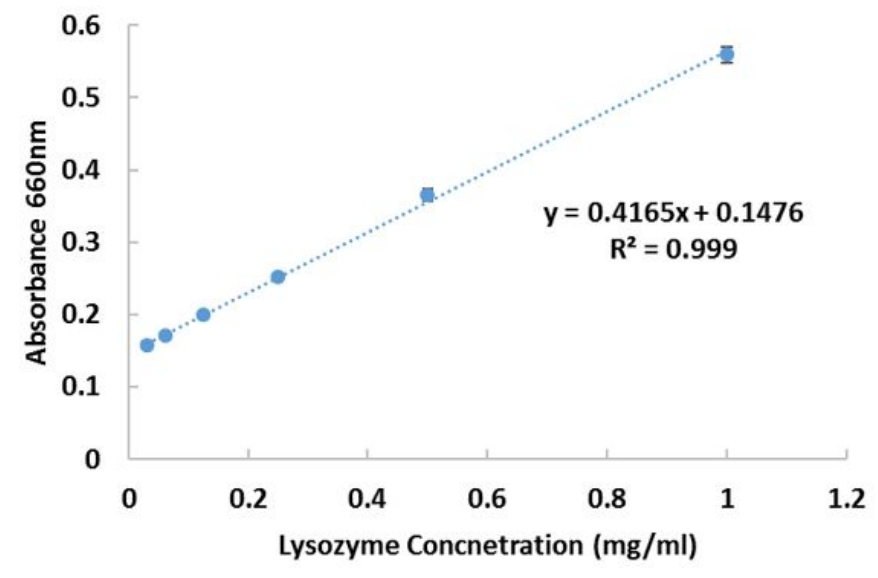

B. RFU vs Protein Concentration standard curve

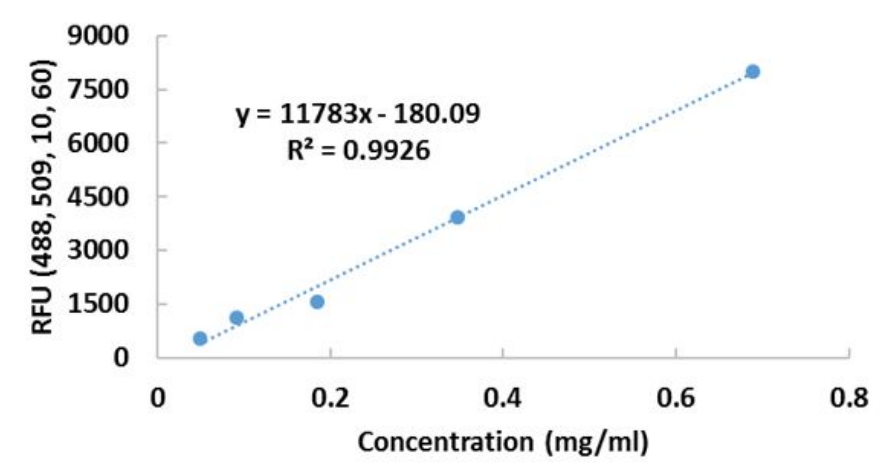

Supplement Figure 1 - (A) Standard curve of known protein concentration of the lysozyme solution and absorbance at 660nm. (B) Standard curve of protein concentration and relative fluorescence of sfGFP. Excitation at $488 \mathrm{~nm}$, emission at $509 \mathrm{~nm}$ with bandwidth of $10 \mathrm{~nm}$, and gain at 60 .

\section{Supplement 2 - Comparing Aerobically and Anaerobically}

\section{cultured Lysates.}

Throughout this paper, lysates capable of Anaerobic Fermentation (Evans $\mathrm{NH}_{4}{ }^{+}$- no air) or Anaerobic Respiration (Evans $\mathrm{NO}_{3}{ }^{-}-$no air) have been compared. A control lysate was generated by culturing cells in minimal media (Evans), and supplementing it with glucose, nitrate and ammonium at $40 \mathrm{mM}$ concentration. This lysate was used to setup CFPS reactions side by side with AR and AF lysates. Throughout this project, we observed that without supplementation AR lysate always performed worse than the AF lysate. This was attributed to the fact that the AR cells were starved of the reduced nitrogen source (ammonium) during the growth, as such, they were "stressed", and performed worse during the CFPS experiments. To address this a control lysate was generated. Previously in the literature it was demonstrated that even supplementation of moderate ammonium in addition to nitrate will not inhibit cells from expressing the proteins of the Nar anaerobic nitrogen metabolism.

When comparing three lysates we observed that the unsuplemented control lysate performed better than the AR lysate. This supported the hypothesis that supplementation of ammonium to growth media 
partially alleviates nutritional stress, thereby resulting in a more productive CFPS lysate. However, the control lysate, supplemented with nitrite during CFPS did not cope with the supplement as well as the AR lysate. This was attributed to the fact that supplementation of ammonium during growth potentially prevents cells from expressing the same concentration of Nar pathway proteins complexes compared to the AR lysate. This experiment was performed in duplicates.

Supplement Table 2 - Relative Fluorescence Yield (total amount of SfGFP expressed during CFPS reaction) of various aerobic and anaerobic lysates. Rate of change of relative fluorescence (initial protein expression rate) during the linear phase of the CFPS reaction. Linear phase was defined as a fixed time window (30 minutes) - 20 minutes after the reaction started and before 50 minutes.

\begin{tabular}{|c|c|c|c|c|c|}
\hline & $\begin{array}{l}\text { Lysate } \\
\end{array}$ & Trial 1 & Trial 2 & AVG & STDEV \\
\hline \multirow{4}{*}{ RFU Yield } & Evans $\mathrm{NH}_{4}^{+}$(no air) & 4770.00 & 4842.00 & 4806.00 & 50.91 \\
\hline & 2xYTPG (air) & 3191.00 & 3139.00 & 3165.00 & 36.77 \\
\hline & Evans $\mathrm{NO}_{3}^{-}$(no air) & 2135.00 & 2111.00 & 2123.00 & 16.97 \\
\hline & Evans $\mathrm{NH}_{4}^{+}$(air) & 1811.00 & 1761.00 & 1786.00 & 35.36 \\
\hline \multirow{4}{*}{$\begin{array}{c}\text { Rate } \\
\text { (RFU/min) }\end{array}$} & Evans $\mathrm{NH}_{4}^{+}$(no air) & 79.77 & 81.42 & 80.60 & 1.16 \\
\hline & 2xYTPG (air) & 42.41 & 41.87 & 42.14 & 0.39 \\
\hline & Evans $\mathrm{NO}_{3}^{-}$(no air) & 31.64 & 31.48 & 31.56 & 0.11 \\
\hline & Evans $\mathrm{NH}_{4}^{+}$(air) & 20.53 & 20.25 & 20.39 & 0.20 \\
\hline
\end{tabular}

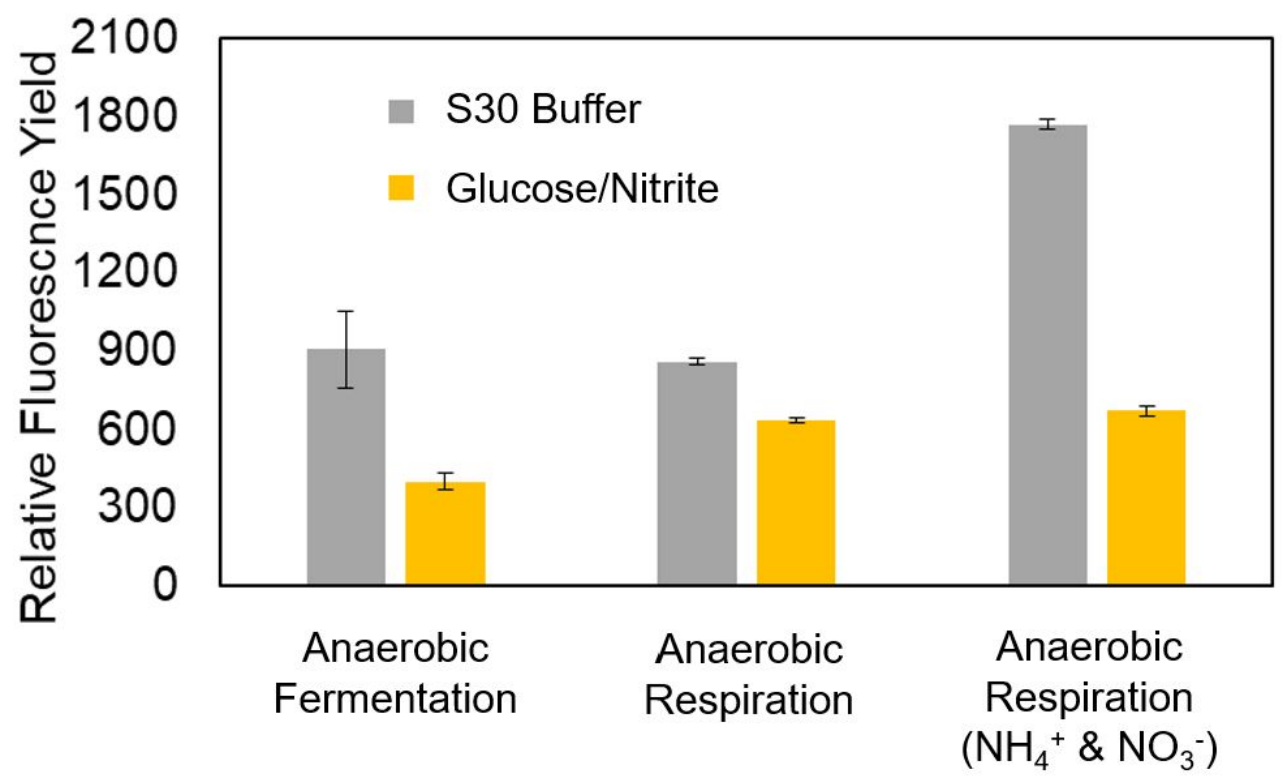

Supplement Figure 2 - Relative fluorescence yield (fluorescence reading at the end of the reaction normalized by background fluorescence) measured from various cell lysates. CFPS reactions were composed with E. coli lysate that was conditioned to engage in either fermentation, Anaerobic Respiration or both. The aforementioned CFPS reactions were either supplemented with $40 \mathrm{mM}$ glucose and $40 \mathrm{mM} \mathrm{NO}_{2}^{-}$("Glucose/Nitrite") or 530 reaction buffer ("S30 Buffer"). All error bars represent the $95 \%$ confidence interval. 


\section{Supplement 3 -Nitrite supplement optimization.}

In this paper we attempted to identify the optimal concentration of nitrite supplementation to CFPS reactions. Nitrite acted as an alternative terminal electron acceptor. High concentrations of nitrite while provided some benefit to the AR lysate, overall decreased the efficiency of protein expression in CFPS reactions. As such, we attempted to identify an optimal concentration of the supplement. The gradient experiment was performed in duplicates twice. The data from Supplement Tables 3 and 4 were presented in the main manuscript. Optimal concentration of the nitrite supplement was identified to be below $40 \mathrm{mM}$ and above $10 \mathrm{mM}$. The glucose concentration gradient, as well as nitrate concentration gradient is described in the Supplement Section 7.

Supplement Table 3 - raw RFU values of CFPS reactions (after 90min) with various concentrations of nitrite supplement, and fixes concentration of Glucose (40mM). Anaerobic Respiration (AR) capable lysate. This is data used in Figure 3 of the main manuscript.

\begin{tabular}{|cccccc|}
\hline \multicolumn{6}{c|}{ Anaerobic Respiration Lysate } \\
[Nitrite $(\mathbf{m M})$ ] & Trial 1 & Trial 2 & Average & Stdev & $\mathbf{9 9 \%}$ \\
\hline 0 & 2928 & 2805 & 2866.5 & 87.0 & 173.9 \\
1 & 2661 & 2541 & 2601 & 84.9 & 169.7 \\
5 & 2974 & 2947 & 2960.5 & 19.1 & 38.2 \\
10 & 3264 & 3356 & 3310 & 65.1 & 130.1 \\
20 & 3357 & 891 & 2124 & 1743.7 & 3487.5 \\
30 & 3242 & 3089 & 3165.5 & 108.2 & 216.4 \\
40 & 2432 & 2565 & 2498.5 & 94.0 & 188.1 \\
80 & 1765 & 1743 & 1754 & 15.6 & 31.1 \\
\hline
\end{tabular}

Supplement Table 4 - raw RFU values of CFPS reactions (after 90min) with various concentrations of nitrite supplement, and fixes concentration of Glucose (40mM). Anaerobic Fermentation (AF) capable lysate. This is data used in Figure 3 of the main manuscript.

\begin{tabular}{|cccccc|}
\hline \multicolumn{6}{c}{ Anaerobic Fermentation Lysate } \\
[Nitrite (mM)] & Trial 1 & Trial 2 & Average & Stdev & $\mathbf{9 9 \%}$ \\
\hline 0 & 5889 & 5810 & 5849.5 & 55.9 & 111.7 \\
1 & 5149 & 5360 & 5254.5 & 149.2 & 298.4 \\
5 & 4741 & 4757 & 4749 & 11.3 & 22.6 \\
10 & 3639 & 3735 & 3687 & 67.9 & 135.8 \\
20 & 2940 & 2937 & 2938.5 & 2.1 & 4.2 \\
30 & 2815 & 2853 & 2834 & 26.9 & 53.7 \\
40 & 2180 & 2255 & 2217.5 & 53.0 & 106.1 \\
80 & 478 & 1950 & 1214 & 1040.9 & 2081.7 \\
\hline
\end{tabular}


Supplement Table 5 - raw RFU values of CFPS reactions (after 90min) with various concentrations of nitrite supplement, and fixes concentration of Glucose (4OmM). Anaerobic Respiration (AR) capable lysate. This is a second trial of this experiment, and the data was not used in the main manuscript

\begin{tabular}{|c|c|c|c|c|c|}
\hline \multicolumn{7}{c|}{ Anaerobic Respiration Lysate } \\
[Nitrite (mM)] & Trial 1 & Trial 2 & Average & Stdev & $\mathbf{9 9 \%}$ \\
\hline 40 & 3392 & 2581 & 2986.5 & 108.2 & 216.4 \\
\hline 30 & 4084 & 3626 & 3855 & 1743.7 & 3487.5 \\
\hline 20 & 4228 & 3573 & 3900.5 & 65.1 & 130.1 \\
\hline 10 & 3057 & 2588 & 2822.5 & 19.1 & 38.2 \\
\hline 5 & 3459 & 2196 & 2827.5 & 84.9 & 169.7 \\
\hline 0 & 2840 & 2981 & 2910.5 & 87.0 & 173.9 \\
\hline
\end{tabular}

Supplement Table 6 - raw RFU values of CFPS reactions (after 90min) with various concentrations of nitrite supplement, and fixes concentration of Glucose (40mM). Anaerobic Respiration (AR) capable lysate. This is a second trial of this experiment, and the data was not used in the main manuscript

\begin{tabular}{|c|c|c|c|c|c|}
\hline \multicolumn{7}{c}{ Anaerobic Fermentation Lysate } \\
[Nitrite (mM)] & Trial 1 & Trial 2 & Average & Stdev & $\mathbf{9 9 \%}$ \\
\hline 40 & 973 & 547 & 760 & 301.2 & 602.5 \\
\hline 30 & 2549 & 1688 & 2118.5 & 608.8 & 1217.6 \\
\hline 20 & 3457 & 2319 & 2888 & 804.7 & 1609.4 \\
\hline 10 & 3697 & 2472 & 3084.5 & 866.2 & 1732.4 \\
\hline 5 & 4164 & 3091 & 3627.5 & 758.7 & 1517.5 \\
\hline 0 & 4359 & 3242 & 3800.5 & 789.8 & 1579.7 \\
\hline
\end{tabular}

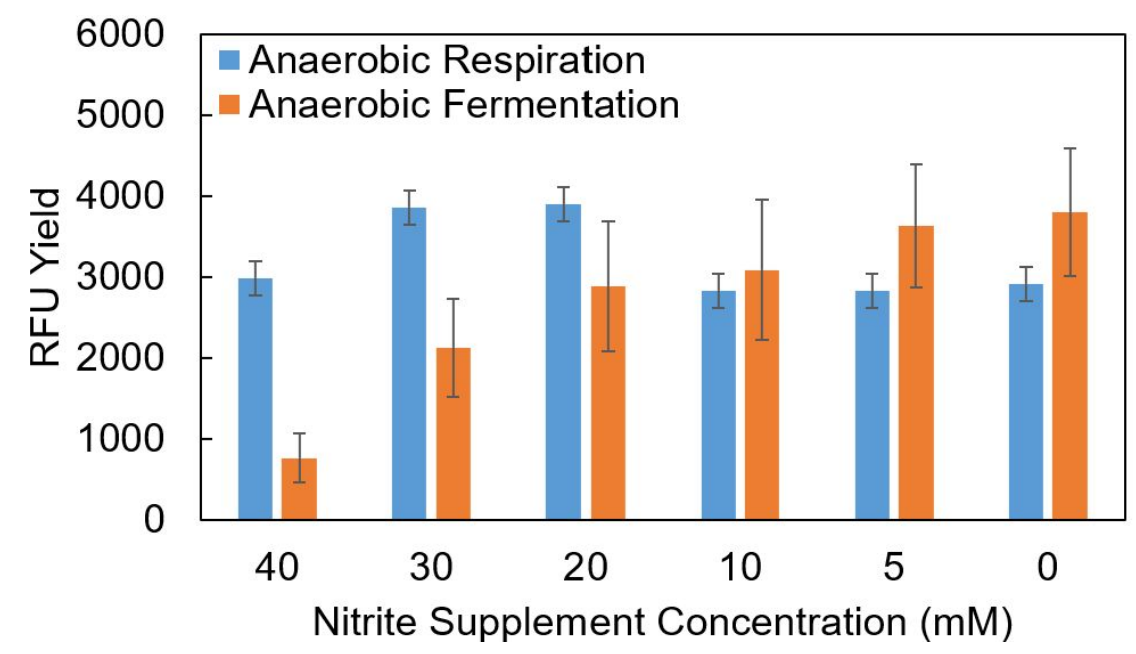

Supplement Figure 3 - Relative fluorescence Units CFPS reactions (after 90min) with various concentrations of nitrite supplement, and fixes concentration of Glucose (40mM). Anaerobic Respiration (AR) capable lysate. This is a second trial of this experiment (supplement tables 5 and 6), and the data was not used in the main manuscript

After identifying a range of optimal nitrite supplement concentrations, we repeated the experiment described in Supplement Section 2. The results demonstrated that the control lysate did not express 
proteins of the Nar pathway as well as the AR lysate. Once again, supplementation of $10 \mathrm{mM}$ and 20 $\mathrm{mM}$ nitrite decreased the efficiency of the AF lysate (as well as the aerobically prepared lysates, blue and grey respectively) but increased the efficiency of the AR lysate (red). Control reactions (void) were performed without glucose or nitrite, and had inconsistent effects on the performance of CFPS reactions. The investigation of the underlying mechanisms guiding the performance of CFPS reactions in the void condition are outside of the scope of this work, as they do not conflict with our findings.

Supplement Table 7-Raw RFU values of CFPS reactions (after $90 \mathrm{~min}$ ) with optimal and non-optimal concentrations of nitrate, and with or without glucose. The data was not used in the main manuscript.

\begin{tabular}{|c|c|c|c|c|c|}
\hline Supplement & Lysate & Trial 1 & Trial 2 & AVG & STDEV \\
\hline \multirow{4}{*}{ 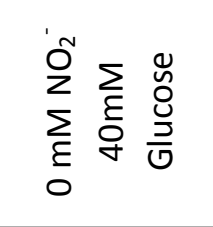 } & $\mathrm{NH}_{4}^{+}$(air) & 2199 & 2206 & 2202.5 & 4.9 \\
\hline & $\mathrm{NH}_{4}^{+}$(no air) & 6207 & 6161 & 6184 & 32.5 \\
\hline & $\mathrm{NO}_{3}^{-}$(no air) & 2384 & 2405 & 2394.5 & 14.8 \\
\hline & $\mathrm{NH}_{4}^{+} \& \mathrm{NO}_{3}^{-}$(no air) & 6293 & 6084 & 6188.5 & 147.8 \\
\hline \multirow{4}{*}{ 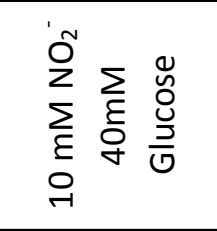 } & $\mathrm{NH}_{4}^{+}$(air) & 2000 & 2007 & 2003.5 & 4.9 \\
\hline & $\mathrm{NH}_{4}^{+}$(no air) & 4268 & 4219 & 4243.5 & 34.6 \\
\hline & $\mathrm{NO}_{3}^{-}$(no air) & 3464 & 3445 & 3454.5 & 13.4 \\
\hline & $\mathrm{NH}_{4}^{+} \& \mathrm{NO}_{3}^{-}$(no air) & 4582 & 4625 & 4603.5 & 30.4 \\
\hline \multirow{4}{*}{ 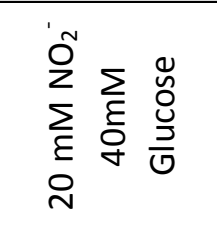 } & $\mathrm{NH}_{4}^{+}$(air) & 1623 & 1657 & 1640 & 24.0 \\
\hline & $\mathrm{NH}_{4}^{+}$(no air) & 3518 & 3526 & 3522 & 5.7 \\
\hline & $\mathrm{NO}_{3}^{-}$(no air) & 3226 & 3255 & 3240.5 & 20.5 \\
\hline & $\mathrm{NH}_{4}^{+} \& \mathrm{NO}_{3}^{-}$(no air) & 3204 & 3232 & 3218 & 19.8 \\
\hline \multirow{4}{*}{ 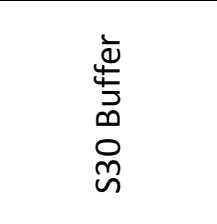 } & $\mathrm{NH}_{4}^{+}$(air) & 2672 & 2797 & 2734.5 & 88.4 \\
\hline & $\mathrm{NH}_{4}^{+}$(no air) & 5201 & 4956 & 5078.5 & 173.2 \\
\hline & $\mathrm{NO}_{3}^{-}$(no air) & 5128 & 4948 & 5038 & 127.3 \\
\hline & $\mathrm{NH}_{4}^{+} \& \mathrm{NO}_{3}^{-}$(no air) & 3253 & 3241 & 3247 & 8.5 \\
\hline
\end{tabular}




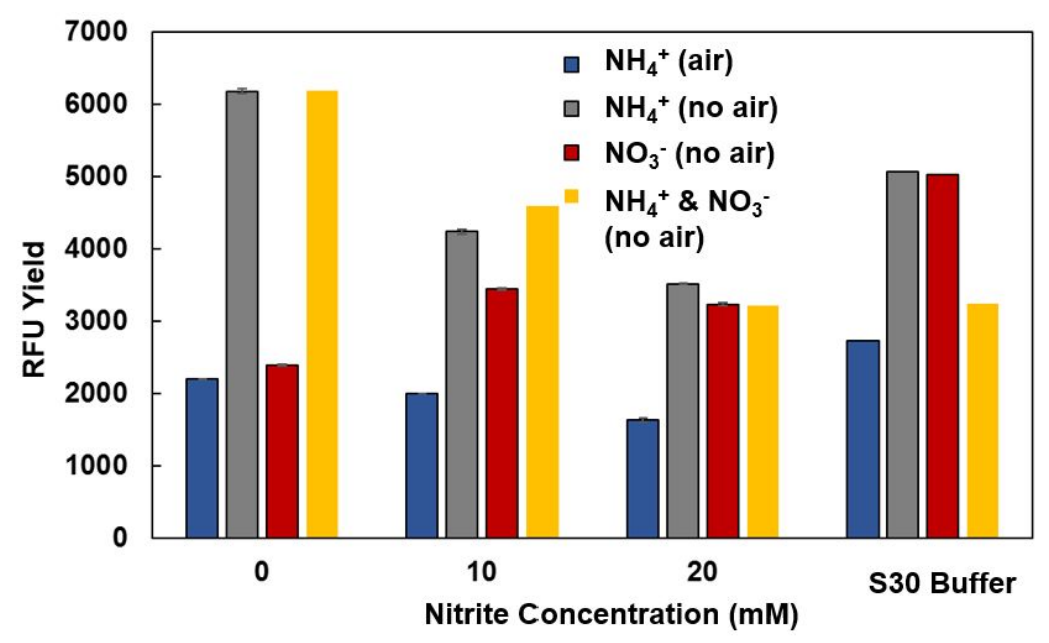

Supplement Figure 4-Relative Fluorescence (RFU) Yield of CFPS reactions (after $90 \mathrm{~min}$ ) with optimal and non-optimal concentrations of nitrite, and with or without glucose as supplements. Negative control, was supplemented with 530 buffer (no supplements). The data was not used in the main manuscript (Supplement Table 7).

\section{Supplement 4 - NirB knockout experiments}

In this work, we also investigated what protein complex of the Nar anaerobic nitrogen metabolism pathway plays a central role in increasing the efficiency of the AR lysate. We suspect that soluble NirB protein of the Nir complex reduces nitrite to ammonium and concurrently oxidizes NADH. This process also contributes to more efficient oxidation of glucose as described previously.

To test that, we acquired E. coli lysate from K12 (wild type) and K12 NirB knockout cells. Since the expression yield was very low, as a control we setup another CFPS reaction from K12 lysate with S30 buffer instead of DNA to measure autofluorescence, and ensure that the fluorescence signal from experimental conditions is above the baseline of the control (buffer condition). The fluorescence from the control condition was subtracted from the fluorescence of the DNA containing reactions. The results clearly indicate that the supplementation of nitrite at an optimal concentration of $20 \mathrm{mM}$ increase the productivity of both NirB knockout and the wild type (K12). The productivity of the wild type lysate increased significantly more than the productivity of the knockout lysate. The increase in productivity of the knockout lysate was attributed to the fact that the knockout lysate was potentially expressing proteins of the Nap pathway to overcompensate for the lack of the Nar pathway. This further indicated that there is a variety of anaerobic nitrogen (or other terminal electron acceptor) pathways that can increase the productivity of protein expression. 
Supplement Table 8-raw RFU values of CFPS reactions (after 90min) either supplemented with glucose (40mM) and nitrite (20mM) or not ("S30 Buffer"). Control experiment consisted of CFPS reaction without DNA (autofluorescence).

\begin{tabular}{|lcccccc|}
\hline \multicolumn{1}{|c}{ Strain } & \multicolumn{2}{c}{ Raw Replicates } & \multicolumn{2}{c}{ Normalized } & AVG & STDEV \\
\hline K12 (Glucose/Nitrite) & 374 & 388 & 354 & 365 & 359.5 & 11.7 \\
NirB- (Glucose/Nitrite) & 142 & 145 & 122 & 122 & 122 & 0.0 \\
K12 (S30 Buffer) & 29 & 27 & 9 & 4 & 6.5 & 5.3 \\
NirB- (S30 Buffer) & 52 & 49 & 32 & 26 & 29 & 6.4 \\
Negative Control & 20 & 23 & 0 & 0 & 0 & 0.0 \\
\hline
\end{tabular}

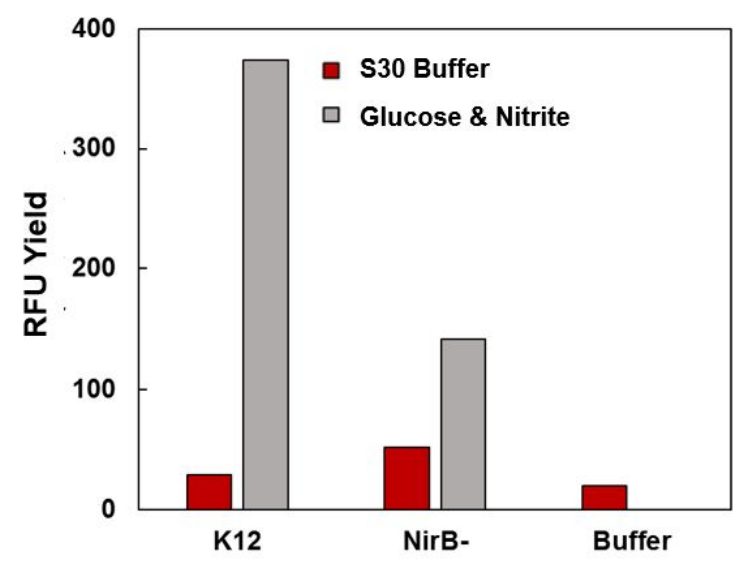

Supplement Figure 5 - Relative fluorescence (RFU) of CFPS reactions (after 90min) either supplemented with glucose (40mM) and nitrite (20mM) or not ("S3O Buffer"). Control experiment consisted of CFPS reaction without DNA (autofluorescence) "Buffer". This data is from the Supplement Table 8.

\section{Supplement 5 - Anaerobic (glove box) CFPS reactions}

We suspected that the most advantage of the AR lysate can be gained in the anaerobic conditions, when there are absolutely no traces of dissolved oxygen that can be used as a terminal electron acceptor. To test that, we performed experiments under completely anaerobic conditions, in a "glove box" with 95\% nitrogen and $5 \%$ hydrogen gas mixture replacing air.

It was previously determined that the AR lysate performs best when it is supplemented with $10-30 \mathrm{mM}$ nitrite and $40 \mathrm{mM}$ glucose. We used optimal $(20 \mathrm{mM})$ nitrite supplement concentration for this experiment. Individual components of the CFPS reactions (DNA, master mix, lysate, S30 buffer and supplements) were aliquoted in the individual containers, degassed using the Airlock, and then assembled in the glove box. These CFPS reactions were conducted at room temperature in $1.5 \mathrm{ml}$ tubes, and were covered in metal foil to avoid photodegradation. After, 8 hours of incubation, the tubes were removed from the airlock, and placed in the plate reader for incubation at $37 \mathrm{C}$ and concurrent folding (oxygen dependent maturation of sfGFP). The data in the supplement table 9 was used to generate Figure 5 that demonstrated a significant gain in performance when the AR lysate CFPS reactions were supplemented with nitrite. 
Supplement Table 9 - raw RFU values of CFPS reactions (after glove box incubation and sfGFP folding in the plate reader) either supplemented with glucose $(40 \mathrm{mM})$ and nitrite $(20 \mathrm{mM})$ or not supplemented (void volume filled with 530 buffer). This is the data used in the main manuscript, Figure 5.

\begin{tabular}{|ccccccc|}
\hline Lysate & Supplementation & Replicate 1 & Replicate 2 & AVG & STDEV \\
\hline Evans $\mathrm{NH}_{4}^{+}$(air) & No & 1132 & 968 & 1050 & 116.0 \\
Evans $\mathrm{NH}_{4}^{+}$(air) & Yes & 326 & 170 & 248 & 110.3 \\
Evans $\mathrm{NH}_{4}^{+}$(no air) & No & 600 & 1006 & 803 & 287.1 \\
Evans $\mathrm{NH}_{4}^{+}$(no air) & Yes & 396 & 199 & 297.5 & 139.3 \\
Evans $\mathrm{NO}_{3}^{-}$(no air) & No & 82 & 100 & 91 & 12.7 \\
${\text { Evans } \mathrm{NO}_{3}^{-} \text {(no air) }}_{\text {Evans } \mathrm{NH}_{4}^{+}{ }^{+} \mathrm{NO}_{3}^{-} \text {(no air) }}$ & Yes & 225 & 355 & 290 & 91.9 \\
Evans $\mathrm{NH}_{4}^{+}$\& $\mathrm{NO}_{3}^{-}$(no air) & No & 2092 & 1885 & 1988.5 & 146.4 \\
\hline
\end{tabular}

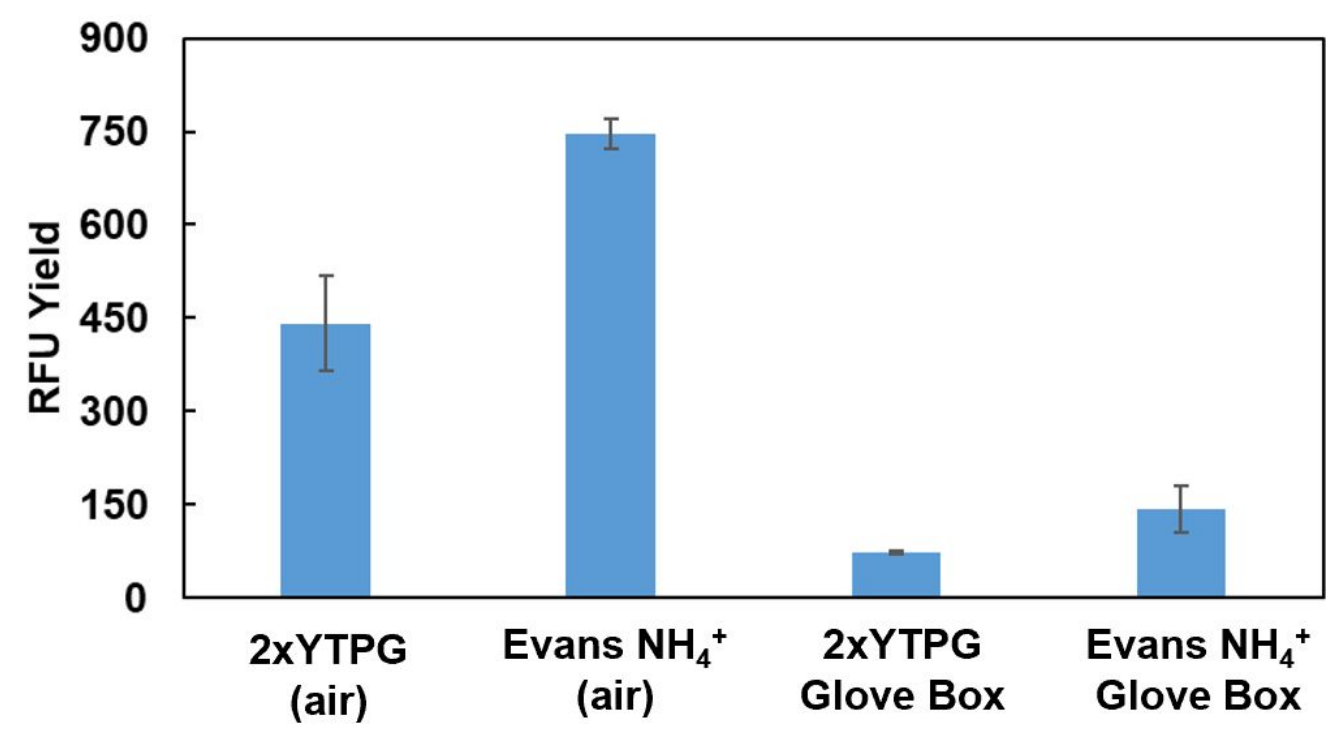

Supplement Figure 6 - Fluorescence yield of CFPS reactions performed at room temperature in aerobic conditions (atmospheric oxygen concentration) and anaerobic (glove box) conditions. Data from supplement Table 10.

Supplement Table 10 -- Fluorescence yield data of CFPS reactions performed at room temperature in aerobic conditions (atmospheric oxygen concentration) and anaerobic (glove box) conditions. Data from supplement figure 6.

\begin{tabular}{|c|c|c|c|c|c|}
\hline Lysate & Replicate 1 & Replicate 2 & Replicate 3 & AVG & STDEV \\
\hline 2xYTPG - Air & 515 & 364 & 445 & 441.3 & 75.6 \\
\hline Evans $\mathrm{NH}_{4}^{+}$(no air) - Air & 718 & 761 & 758 & 745.7 & 24.0 \\
\hline 2xYTPG - Glove Box & 75 & 74 & 69 & 72.7 & 3.2 \\
\hline Evans $\mathrm{NH}_{4}^{+}$(no air) - Glove Box & 181 & 142 & 106 & 143.0 & 37.5 \\
\hline
\end{tabular}




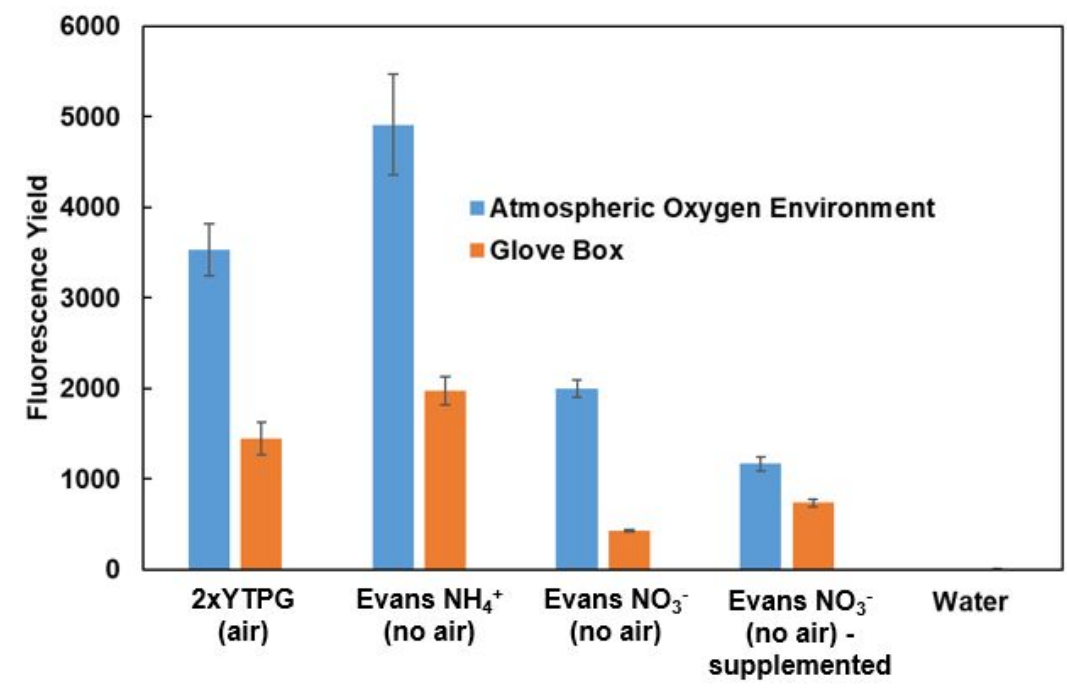

Supplement Figure 7-Fluorescence yield of CFPS reactions performed at room temperature in aerobic conditions (atmospheric oxygen concentration) and anaerobic (glove box) conditions. Data from supplement Table 11

Supplement Table 11 -- Fluorescence yield of CFPS reactions performed at room temperature in aerobic conditions (atmospheric oxygen concentration) and anaerobic (glove box) conditions. Data from supplement Figure 7.

\begin{tabular}{|c|c|c|c|c|c|c|c|c|c|c|}
\hline \multirow{2}{*}{ Lysate } & \multicolumn{5}{|c|}{ Atmospheric Oxygen Environment } & \multicolumn{5}{|c|}{ Glove Box Environment } \\
\hline & Trial 1 & Trial 2 & Trial 3 & AVG & STDEV & Trial 1 & Trial 2 & Trial 3 & AVG & STDEV \\
\hline 2xYTPG & 3216 & 3578 & 3785 & 3526.3 & 288.0 & 1258 & 1609 & 1484 & 1450.3 & 177.9 \\
\hline Evans $\mathrm{NH}_{4}{ }^{+}$(no air) & 4349 & 4928 & 5457 & 4911.3 & 554.2 & 1797 & 2056 & 2072 & 1975.0 & 154.4 \\
\hline Evans $\mathrm{NO}_{3}^{-}$(no air) & 1888 & 2048 & 2059 & 1998.3 & 95.7 & 441 & 412 & 438 & 430.3 & 15.9 \\
\hline $\begin{array}{c}\text { Evans } \mathrm{NO}_{3}^{-} \text {(no air) } \\
\text { supplemented }\end{array}$ & 1082 & 1202 & 1213 & 1165.7 & 72.7 & 745 & 765 & 694 & 734.7 & 36.6 \\
\hline Control (no DNA) & & & & & & 12 & 12 & 13 & 12.3 & 0.6 \\
\hline
\end{tabular}




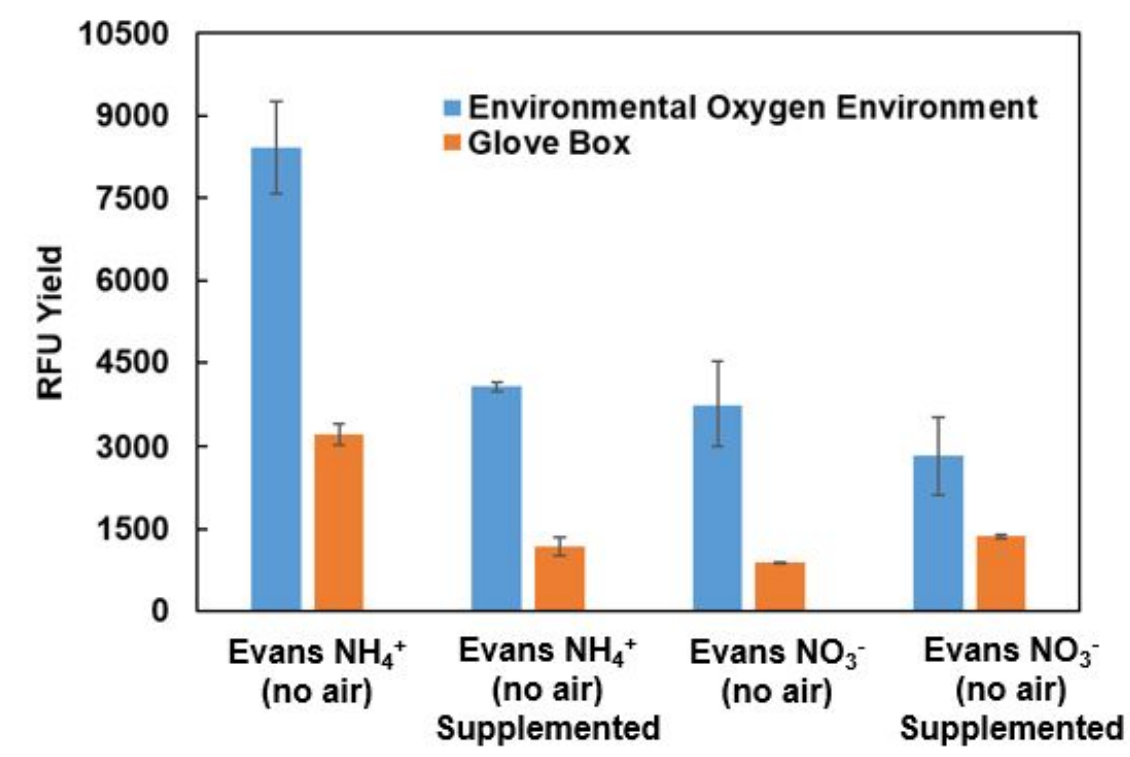

Supplement Figure 8- Fluorescence yield of CFPS reactions performed at room temperature in aerobic conditions (atmospheric oxygen concentration) and anaerobic (glove box) conditions. Data from supplement Table 12

Supplement Table 12 - Fluorescence yield of CFPS reactions performed at room temperature in aerobic conditions (atmospheric oxygen concentration) and anaerobic (glove box) conditions. Data from supplement figure 8.

\begin{tabular}{|c|c|c|c|c|c|c|c|c|}
\hline \multirow{2}{*}{ Lysate } & \multicolumn{4}{|c|}{ Atmospheric Oxygen Environment } & \multicolumn{4}{|c|}{ Glove Box Environment } \\
\hline & Replicat & eplicate 2 & AVG & STDEV & Replicate & 1 Replicate 2 & AVG & STDEV \\
\hline $\begin{array}{c}\text { Evans } \mathrm{NH}_{4}^{+} \\
\text {(no air) }\end{array}$ & 9020 & 7824 & 8422 & 845.7 & 3357 & 3087 & 3222 & 190.9 \\
\hline $\begin{array}{c}\text { Evans } \mathrm{NH}_{4}^{+} \\
\text {(no air) } \\
\text { Supplemented }\end{array}$ & 3995 & 4119 & 4057 & 87.7 & 1292 & 1053 & 1172.5 & 169.0 \\
\hline $\begin{array}{c}\text { Evans } \mathrm{NO}_{3}^{-} \\
\text {(no air) }\end{array}$ & 4292 & 3220 & 3756 & 758.0 & 901 & 893 & 897 & 5.7 \\
\hline $\begin{array}{c}\text { Evans } \mathrm{NO}_{3}^{-} \\
\text {(no air) } \\
\text { Supplemented }\end{array}$ & 2334 & 3331 & 2832.5 & 705.0 & 1339 & 1393 & 1366 & 38.2 \\
\hline
\end{tabular}

\section{Supplement 6 - Relative loss or gain calculations and error}

\section{propagation.}

In order to demonstrate the advantage of using the AR lysate over the AF lysate in a cleaner way, we calculated the percentage gain (or loss) for various CFPS reactions when comparing unsupplemented vs supplemented conditions. This was calculated by taking the fluorescence yield of the supplemented version of the CFPS condition and dividing it by the unsupplemented version of that CFPS reaction. Then 
subtracting $100 \%$ resulted in a percentage loss or gain in performance. The exact conditions that were used for this calculation are presented in the supplement table 13.

Supplement Table 13 -- Relative gain and loss of performance values used in the Figure 5 of the main manuscript. Data summarized from Supplement Table 7 (atmospheric oxygen), and Supplement Table 9 (glove box).

\begin{tabular}{|c|c|c|c|c|c|c|c|c|}
\hline Lysate & Glove Box & Supplementation & Trial 1 & Trial 2 & AVG & STDEV & Ratio & Gain (\%) \\
\hline Evans $\mathrm{NH}_{4}^{+}$ & No & No & 6207 & 6161 & 6184 & 32.5 & & \\
\hline (No Air) & No & Yes & 3518 & 3526 & 3522 & 5.7 & 0.57 & -43 \\
\hline Evans $\mathrm{NO}_{3}^{-}$ & No & No & 2384 & 2405 & 2394.5 & 14.8 & & \\
\hline (No Air) & No & Yes & 3226 & 3255 & 3240.5 & 20.5 & 1.35 & 35 \\
\hline Evans $\mathrm{NH}_{4}^{+}$ & Yes & No & 600 & 1006 & 803 & 287.1 & & \\
\hline (No Air) & Yes & Yes & 396 & 199 & 297.5 & 139.3 & 0.37 & -63 \\
\hline Evans $\mathrm{NO}_{3}^{-}$ & Yes & No & 82 & 100 & 91 & 12.7 & & \\
\hline (No Air) & Yes & Yes & 225 & 355 & 290 & 91.9 & 3.19 & 219 \\
\hline
\end{tabular}

Supplement Table 14 - Error propagation. Variance is calculated based on equation 2. Sigma is calculated based on equation 5 , and represents $68^{\text {th }}$ percentile confidence interval. Data in the main manuscript was reported in percent values, and, therefore, the error was normalized to the same units as well ("Error Bars (\%)" column of the table).

\begin{tabular}{|c|cccc|c|}
\hline Lysate & Condition & AVG & Variance & Sigma $\mathbf{( 6 8}^{\text {th }}$ ) & $\begin{array}{c}\text { Error Bars } \\
\text { (\%) }\end{array}$ \\
\hline Evans NH $_{4}^{+}$ & Air & 0.570 & $9.811 \mathrm{E}-06$ & 0.003 & 0.313 \\
(No Air) $^{-}$ & Glove Box & 0.370 & $4.764 \mathrm{E}-02$ & 0.218 & 21.826 \\
Evans NO $_{3}{ }^{-}$ & Air & 1.353 & $1.438 \mathrm{E}-04$ & 0.012 & 1.199 \\
(No Air) & Glove Box & 3.187 & $1.219 \mathrm{E}+00$ & 1.104 & 110.412 \\
\hline
\end{tabular}

Error propagation was performed according to methods described in "Data Reduction and Error Analysis for the Physical Sciences" by Philip Bevington and Keith Robinson. First order derivatives were used, and cross terms were dropped as no correlation between error in measurements of unsuplemented and supplemented lysates was observed.

\section{Unsuplemented $=\mathrm{u}$;}

$$
\text { Supplemented }=s \text {; }
$$

(1) Gain (or loss) function $=G(s, u)=\frac{s}{u}$;

(2) $\delta G(s, u)^{2}=\left(\frac{\delta G(s, u)}{\delta s} \delta s\right)^{2}+\left(\frac{\delta G(s, u)}{\delta u} \delta u\right)^{2}$;

(3) $\frac{\delta G(s, u)}{\delta s} \delta s=\frac{1}{u} \delta s$

(4) $\frac{\delta G(s, u)}{\delta u} \delta u=\frac{-u}{u^{2}} \delta u$

(5) $\frac{\delta G(s, u)^{2}}{G(s, u)^{2}}=\left(\frac{\left(\frac{\delta G(s, u)}{\delta s} \delta s\right)^{2}}{s^{2}}+\frac{\left(\frac{\delta G(s, u)}{\delta u} \delta u\right)^{2}}{u^{2}}\right) G(s, u)^{2}$ 


\section{Supplement 7 - Luminescence experiments and ATP}

\section{measurements.}

Up until this point in the paper, our observations suggest that the AR capable lysate is more efficient because it expressed NirB which oxidizes NADH, and as a result of that allows cells to oxidize glucose more efficiently and generate up to $50 \%$ more ATP per molecule of glucose. To elucidate further, we attempted to quantify ATP concentrations in CFPS reactions with AR and AF lysates during the process of CFPS reactions.

We setup CFPS reactions without DNA with AR or AF lysates, and incubated them at $37 \mathrm{C}$ for various durations. Each reaction was conducted in duplicates. Void reactions did not contain the nitrite and glucose supplement at optimal concentrations $(20 \mathrm{mM}$ and $40 \mathrm{mM}$ respectively). To quantify ATP we chose to use Promega's luminescence kit CellTiter-Glo 2.0.

First Trial. During the initial testing we determined that the undiluted CFPS extract contains enough ATP to be detected with the assay without saturation. As such, 1 to 1 concentration of the assay mixture to CFPS reaction mix (in this section CFPS reaction mix refers to the lysate mixed with the master mix, and S30 buffer). The CFPS reactions (50ul each) were incubated for 90 minutes, and fractions were pulled at 30 min increments (Supplement Table 14 \& Supplement Figure 9).

Both void AR and AF lysates demonstrated initial uptick in ATP concentration, followed by exponential decay in ATP concentration. Both supplemented lysates demonstrated a continuous increase in ATP concentration through 90 minutes. It appears that the AR lysate generated slightly higher concentrations of ATP compared to the AF lysate during 30, 60 minutes but not during the 90 minute time points. We expected that eventually, the ATP concentration should decay for both AR and AF lysates. Since this decay was not observed within 120 minutes, we chose to run a second trial for longer (240 minutes). In addition, the assay may have reached the limit of detection at 120 minutes, and in consecutive trials the CFPS lysate was diluted. = 
Supplement Table 14 - raw luminescence experiment readings. Anaerobic Fermentation (Evans media supplemented with glucose and ammonium during growth) and Anaerobic Respiration lysate (Evans media supplemented with glucose and nitrate during growth) CFPS reactions were compared. Reactions were stopped at various time points $-0,30,60,90$ minutes. This is the first trial of this experiment.

\begin{tabular}{|c|c|c|c|c|c|c|}
\hline & Supplement & Lysate & 1 & 2 & AVG & STDEV \\
\hline \multirow{4}{*}{$\begin{array}{l}0 \text { min } \\
\text { (start) }\end{array}$} & \multirow{2}{*}{ S30 Buffer } & Evans $\mathrm{NH}_{4}^{+}$(no air) & 23780 & 19184 & 21482 & 3249.9 \\
\hline & & Evans $\mathrm{NO}_{3}^{-}$(no air) & 22330 & 22383 & 22356.5 & 37.5 \\
\hline & \multirow{2}{*}{ Glucose/Nitrite } & Evans $\mathrm{NH}_{4}^{+}$(no air) & 12480 & 12721 & 12600.5 & 170.4 \\
\hline & & Evans $\mathrm{NO}_{3}^{-}$(no air) & 15903 & 16469 & 16186 & 400.2 \\
\hline \multirow{4}{*}{$\begin{array}{c}30 \mathrm{~min} \\
\text { (start) }\end{array}$} & \multirow{2}{*}{ S30 Buffer } & Evans $\mathrm{NH}_{4}^{+}$(no air) & 24242 & 22822 & 23532 & 1004.1 \\
\hline & & Evans $\mathrm{NO}_{3}^{-}$(no air) & 27732 & 24267 & 25999.5 & 2450.1 \\
\hline & \multirow{2}{*}{ Glucose/Nitrite } & Evans $\mathrm{NH}_{4}^{+}$(no air) & 19031 & 18722 & 18876.5 & 218.5 \\
\hline & & Evans $\mathrm{NO}_{3}^{-}$(no air) & 23134 & 21615 & 22374.5 & 1074.1 \\
\hline \multirow{4}{*}{$\begin{array}{l}60 \mathrm{~min} \\
\text { (start) }\end{array}$} & \multirow{2}{*}{ S30 Buffer } & Evans $\mathrm{NH}_{4}^{+}$(no air) & 9245 & 9021 & 9133 & 158.4 \\
\hline & & Evans $\mathrm{NO}_{3}^{-}$(no air) & 15063 & 14511 & 14787 & 390.3 \\
\hline & \multirow{2}{*}{ Glucose/Nitrite } & Evans $\mathrm{NH}_{4}^{+}$(no air) & 34095 & 32953 & 33524 & 807.5 \\
\hline & & Evans $\mathrm{NO}_{3}^{-}$(no air) & 24802 & 24332 & 24567 & 332.3 \\
\hline \multirow{4}{*}{$\begin{array}{l}90 \text { min } \\
\text { (start) }\end{array}$} & \multirow{2}{*}{ S30 Buffer } & Evans $\mathrm{NH}_{4}^{+}$(no air) & 4376 & 4197 & 4286.5 & 126.6 \\
\hline & & Evans $\mathrm{NO}_{3}^{-}$(no air) & 3949 & 3890 & 3919.5 & 41.7 \\
\hline & \multirow{2}{*}{ Glucose/Nitrite } & Evans $\mathrm{NH}_{4}^{+}$(no air) & 35012 & 34115 & 34563.5 & 634.3 \\
\hline & & Evans $\mathrm{NO}_{3}^{-}$(no air) & 34615 & 34695 & 34655 & 56.6 \\
\hline
\end{tabular}




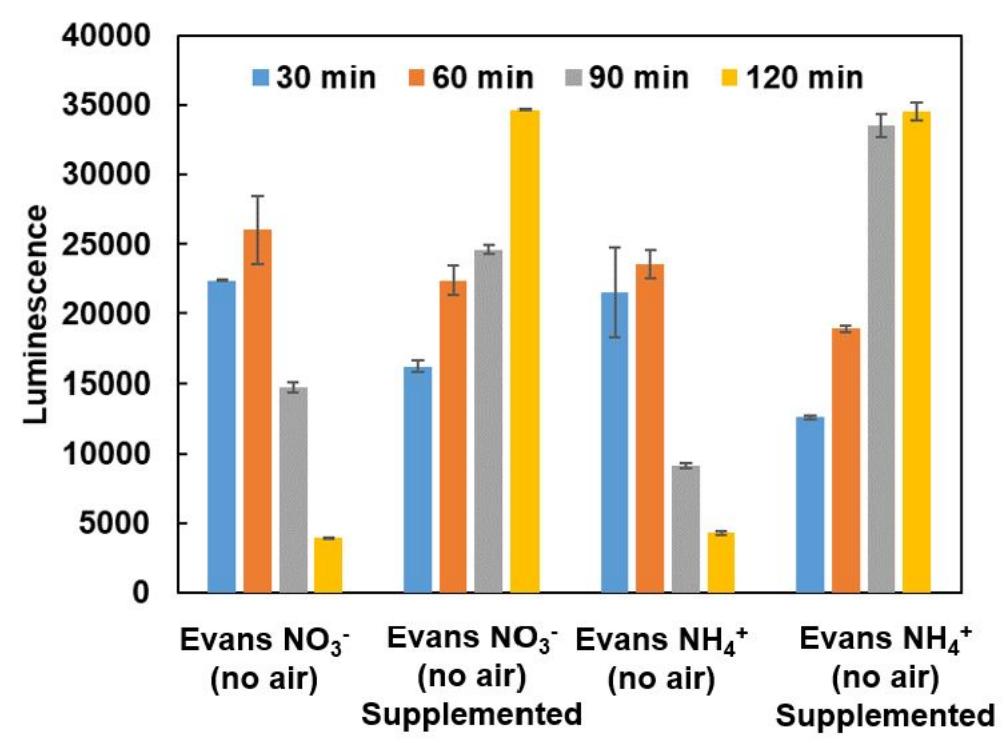

Supplement Figure 9-raw luminescence experiment readings. AF (Evans with ammonium anaerobic) and AR (Evans with nitrate anaerobic) lysate CFPS reactions were compared. Reactions were stopped at various time points - 0, 30, 60, 90 minutes. This is the first trial of this experiment (Supplement Table 14).

Second trial. In this trial, CFPS reactions (50ul each) were diluted 10, 100 and 1000 times with S30 buffer, to make sure that the ATP concentration generated during the CFPS reaction did not exceed the limit of detection. Unlike in the first trial, the CFPS reactions were allowed to develop 37C for up to 240 minutes. In addition, a negative control (NC) was setup from $\mathrm{S} 30$ buffer and the assay mixture to evaluate whether the $1 / 1000 \mathrm{X}$ dilution of the extract generates luminescence signal above the baseline (negative control). The $1 / 100 \mathrm{X}$ dilution was plotted, as it did not saturate the assay, and appeared to generate signal above the baseline of the negative control.

A decrease in ATP concentration was observed at 240 minutes. The same decay of ATP concentrations was observed when both AR or AF lysates were not supplemented with nitrite and glucose. The AR containing CFPS reactions observed a faster decay of ATP. This was attributed to the fact that, as observed previously, the AR lysate is less productive than the AF lysate.

The supplemented AR lysate demonstrated higher ATP concentrations than the AF lysate. However, the difference was small, and since the initial concentration of ATP (at 0 minutes) was higher for the AR lysate than for the AF lysate, the overall higher concentration of ATP at each time point could be attributed to the human error in mixing. Specifically, the master mix contained ATP and PEP. As such, even a slight pipetting error would show up as if the CFPS reaction contained more ATP. 
Supplement Table 15 - raw luminescence experiment readings. AF (Evans with ammonium anaerobic) and AR (Evans with nitrate anaerobic) lysate CFPS reactions were compared ("void" - S30 buffer, "Supl" - Glucose/Nitrite supplemented reactions; NC - negative control, CFPS without DNA). Reactions were stopped at various time points -0, 30, 60, 90 minutes. CFPS reactions were diluted either 10, 100 or 1000 times. See Supplement Section 6 for details. This is the second trial of this experiment.

\begin{tabular}{|c|c|c|c|c|c|c|c|c|c|c|c|c|}
\hline & \multicolumn{12}{|c|}{ Time 0} \\
\hline & \multicolumn{4}{|c|}{$1 / 10 \mathrm{X}$} & \multicolumn{4}{|c|}{$1 / 100 \mathrm{X}$} & \multicolumn{4}{|c|}{$1 / 1000 \mathrm{X}$} \\
\hline & Trial 1 & Trial 2 & AVG & STDEV & Trial 1 & Trial 2 & AVG & STDEV & Trial 1 & Trial 2 & AVG & STDEV \\
\hline AR (void) & 23735 & & & & 19032 & & & & 2863 & & & \\
\hline AR (supl) & 17259 & & & & 19552 & & & & 3761 & & & \\
\hline AF (void) & 25811 & & & & 19621 & & & & 3216 & & & \\
\hline AF (supl) & 12761 & & & & 16339 & & & & 3116 & & & \\
\hline
\end{tabular}

\begin{tabular}{|c|c|c|c|c|c|c|c|c|c|c|c|c|}
\hline & \multicolumn{12}{|c|}{ Time $30 \mathrm{~min}$} \\
\hline & \multicolumn{4}{|c|}{$1 / 10 x$} & \multicolumn{4}{|c|}{$1 / 100 \mathrm{X}$} & \multicolumn{4}{|c|}{$1 / 1000 X$} \\
\hline & Trial 1 & Trial 2 & AVG & STDEV & Trial 1 & Trial 2 & AVG & STDEV & Trial 1 & Trial 2 & AVG & STDEV \\
\hline AR (void) & 32330 & 32339 & 32335 & 6.364 & 14332 & 14228 & 14280 & 73.54 & 2177 & 1944 & 2061 & 164.8 \\
\hline AR (supl) & 22746 & 23001 & 22874 & 180.3 & 18598 & 18089 & 18344 & 359.9 & 3003 & 2692 & 2848 & 219.9 \\
\hline AF (void) & 24345 & 24165 & 24255 & 127.3 & 9569 & 9345 & 9457 & 158.4 & 1840 & 1405 & 1623 & 307.6 \\
\hline AF (supl) & 17709 & 951 & 9330 & 11850 & 805 & 14631 & 7718 & 9776 & 2339 & 2100 & 2220 & 169 \\
\hline
\end{tabular}

\begin{tabular}{|c|c|c|c|c|c|c|c|c|c|c|c|c|}
\hline & \multicolumn{12}{|c|}{ Time 60 min } \\
\hline & \multicolumn{4}{|c|}{$1 / 10 X$} & \multicolumn{4}{|c|}{$1 / 100 \mathrm{X}$} & \multicolumn{4}{|c|}{$1 / 1000 \mathrm{X}$} \\
\hline & Trial 1 & Trial 2 & AVG & STDEV & Trial 1 & Trial 2 & AVG & STDEV & Trial 1 & Trial 2 & AVG & STDEV \\
\hline AR (void) & 15063 & 14132 & 14598 & 658.3 & 7281 & 7034 & 7158 & 174.7 & 1679 & 1332 & 1506 & 245.4 \\
\hline AR (supl) & 24477 & 24343 & 24410 & 94.75 & 24343 & 13370 & 18857 & 7759 & 2961 & 2683 & 2822 & 196.6 \\
\hline AF (void) & 11285 & 11092 & 11189 & 136.5 & 5073 & 4678 & 4876 & 279.3 & 1270 & 943 & 1107 & 231.2 \\
\hline AF (supl) & 29368 & 27445 & 28407 & 1360 & 12414 & 11586 & 12000 & 585.5 & 2581 & 2078 & 2330 & 355.7 \\
\hline
\end{tabular}

\begin{tabular}{|c|c|c|c|c|c|c|c|c|c|c|c|c|}
\hline & \multicolumn{12}{|c|}{ Time 90 min } \\
\hline & \multicolumn{4}{|c|}{$1 / 10 X$} & \multicolumn{4}{|c|}{$1 / 100 \mathrm{X}$} & \multicolumn{4}{|c|}{$1 / 1000 \mathrm{X}$} \\
\hline & Trial 1 & Trial 2 & AVG & STDEV & Trial 1 & Trial 2 & AVG & STDEV & Trial 1 & Trial 2 & AVG & STDEV \\
\hline AR (void) & 6957 & 6670 & 6814 & 202.9 & 3614 & 3533 & 3574 & 57.28 & 685 & 452 & 568.5 & 164.8 \\
\hline AR (supl) & 37611 & 37392 & 37502 & 154.9 & 19191 & 17897 & 18544 & 915 & 4039 & 3393 & 3716 & 456.8 \\
\hline & 6681 & 6505 & 6593 & 124.5 & 4153 & 3536 & 3845 & 436.3 & 794 & 548 & 671 & 173.9 \\
\hline AF (supl) & 38947 & 36595 & 37771 & 1663 & 16534 & 15637 & 16086 & 634.3 & 3577 & 2964 & 3271 & 433.5 \\
\hline
\end{tabular}

\begin{tabular}{|c|c|c|c|c|c|c|c|c|c|c|c|c|}
\hline & \multicolumn{12}{|c|}{ Time $120 \mathrm{~min}$} \\
\hline & \multicolumn{4}{|c|}{$1 / 10 X$} & \multicolumn{4}{|c|}{$1 / 100 \mathrm{X}$} & \multicolumn{4}{|c|}{$1 / 1000 \mathrm{X}$} \\
\hline & Trial 1 & Trial 2 & AVG & STDEV & Trial 1 & Trial 2 & AVG & STDEV & Trial 1 & Trial 2 & AVG & STDEV \\
\hline AR (void) & 3462 & 3480 & 3471 & 12.73 & 2002 & 1769 & 1886 & 164.8 & 407 & 298 & 352.5 & 77.07 \\
\hline AR (supl) & 35914 & 34432 & 35173 & 1048 & 14828 & 15484 & 15156 & 463.9 & 3140 & 2470 & 2805 & 473.8 \\
\hline AF (void) & 5373 & 5216 & 5295 & 111 & 3325 & 2821 & 3073 & 356.4 & 1121 & 743 & 932 & 267.3 \\
\hline AF (supl) & 33449 & 34434 & 33942 & 696.5 & 14329 & 13870 & 14100 & 324.6 & 3031 & 2234 & 2633 & 563.6 \\
\hline
\end{tabular}

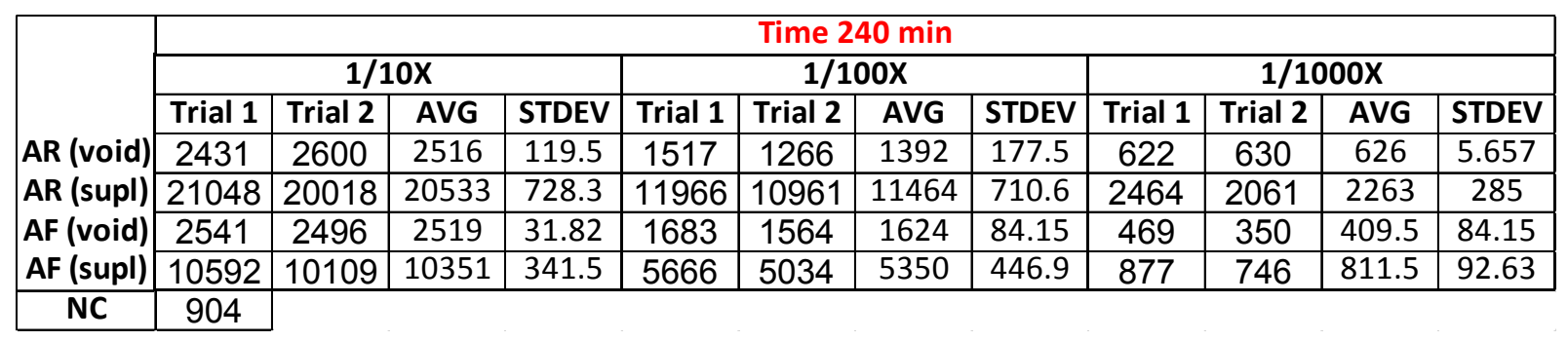




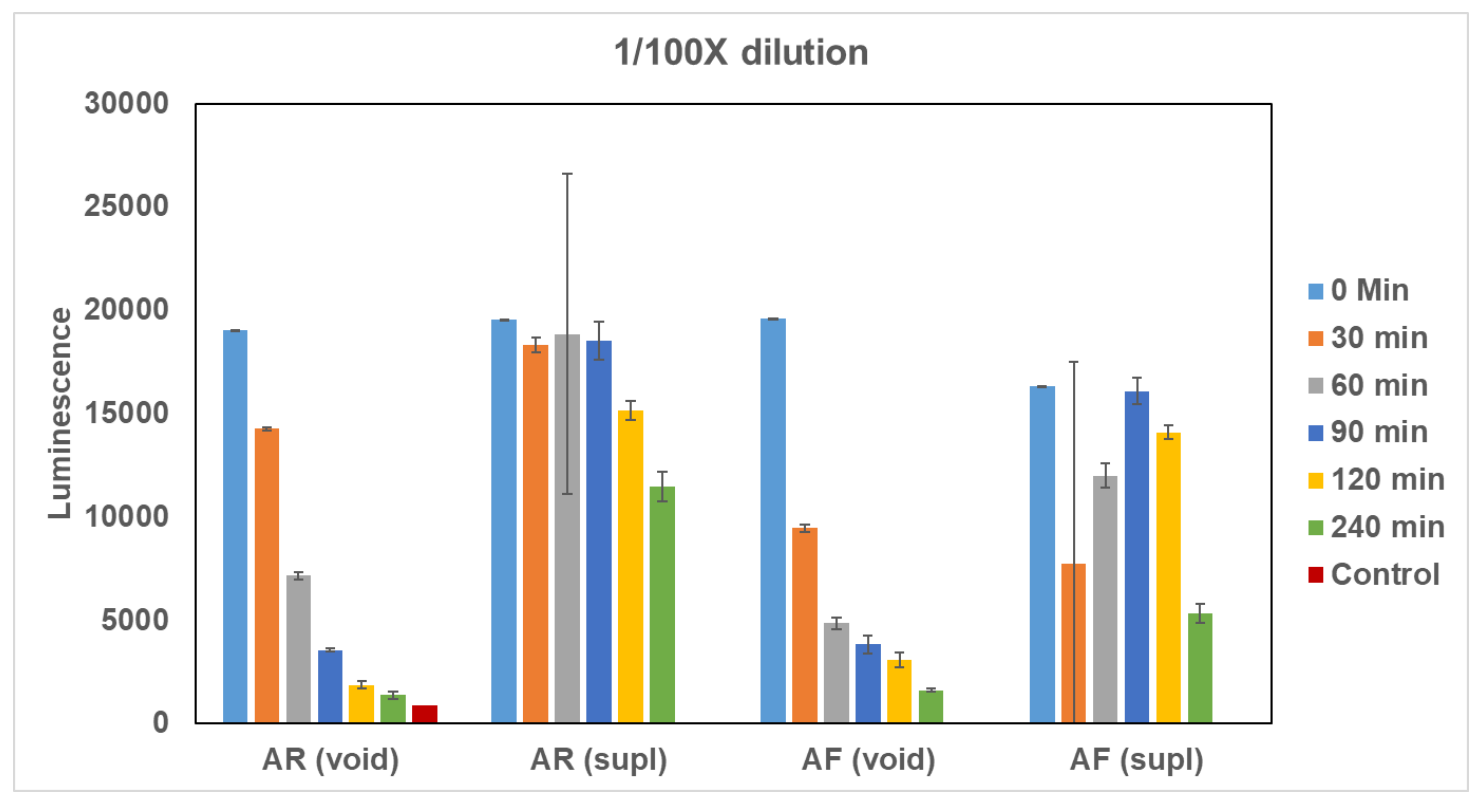

Supplement Figure 10 - raw luminescence experiment readings. AF (Evans with ammonium anaerobic) and AR (Evans with nitrate anaerobic) lysate CFPS reactions were compared ("void" - S30 buffer, "Supl" - Glucose/Nitrite supplemented reactions). Reactions were stopped at various time points -0, 30, 60, 90 minutes. CFPS reactions were diluted either 10, 100 or 1000 times. See Supplement Section 6 for details. This is the second trial of this experiment (Supplement Table 15).

Supplement Table 16 - raw luminescence experiment readings AF (Evans with ammonium anaerobic) and AR (Evans with nitrate anaerobic) lysate CFPS reactions were compared ("void" - S30 buffer, "Supl" - Glucose/Nitrite supplemented reactions).

Reactions were stopped at various time points - 0, 30, 60, 90 minutes. CFPS reactions were diluted 200 times. See Supplement Section 6 for details. "Supplemented" reactions contained both glucose and nitrite. "Glucose only" reactions were supplemented with glucose (40mM) alone. This is the third trial of this experiment. 
0 min

\begin{tabular}{|c|c|c|c|c|c|c|}
\hline & \\
\hline & Trial 1 & Trial 2 & Trial 3 & Trial 4 & AVG & STEV \\
\hline AR (void) & 10899 & 11753 & 11470 & 10899 & 11255.3 & 427.3 \\
\hline AR (glucose only) & 10116 & 10169 & 10670 & 10544 & 10374.8 & 273.9 \\
\hline AR (supplemented) & 10419 & 10520 & 10295 & 10585 & 10454.8 & 126.5 \\
\hline AF (void) & 13526 & 12132 & 12056 & 11985 & 12424.8 & 736.6 \\
\hline AF (glucose only) & 9882 & 8592 & 8719 & 9310 & 9125.8 & 593.4 \\
\hline \multirow[t]{3}{*}{ AF (supplemented) } & 9124 & 9143 & 8925 & 9060 & 9063.0 & 98.6 \\
\hline & \multicolumn{6}{|c|}{$30 \mathrm{~min}$} \\
\hline & Trial 1 & Trial 2 & Trial 3 & Trial 4 & AVG & STEV \\
\hline$A R$ (void) & 9247 & 8826 & 8272 & 8969 & 8828.5 & 410.1 \\
\hline AR (glucose only) & 10145 & 9792 & 10126 & 9920 & 9995.8 & 169.8 \\
\hline AR (supplemented) & 10323 & 10162 & 10093 & 10253 & 10207.8 & 101.0 \\
\hline AF (void) & 9944 & 9604 & 9276 & 8638 & 9365.5 & 556.4 \\
\hline AF (glucose only) & 7317 & 8207 & 7352 & 6924 & 7450.0 & 540.7 \\
\hline AF (supplemented) & 7736 & 7971 & 7577 & 7388 & 7668.0 & 247.1 \\
\hline
\end{tabular}

\begin{tabular}{|c|c|c|c|c|c|c|}
\hline & \multirow{2}{*}{\multicolumn{6}{|c|}{$60 \mathrm{~min}$}} \\
\hline & & & & & & \\
\hline & Trial 1 & Trial 2 & Trial 3 & Trial 4 & AVG & STEV \\
\hline AR (void) & 5366 & 5085 & 4975 & 5055 & 5120.3 & 170.3 \\
\hline AR (glucose only) & 9417 & 9623 & 10288 & 9758 & 9771.5 & 371.8 \\
\hline AR (supplemented) & 10182 & 10430 & 10528 & 10450 & 10397.5 & 149.8 \\
\hline AF (void) & 3727 & 3769 & 3675 & 3593 & 3691.0 & 75.8 \\
\hline AF (glucose only) & 11158 & 10547 & 10894 & 10139 & 10684.5 & 441.4 \\
\hline \multirow[t]{3}{*}{ AF (supplemented) } & 16515 & 16374 & 6891 & 5243 & 11255.8 & 6029.4 \\
\hline & \multicolumn{6}{|c|}{$90 \mathrm{~min}$} \\
\hline & Trial 1 & Trial 2 & Trial 3 & Trial 4 & AVG & STEV \\
\hline AR (void) & 3218 & 3247 & 3209 & 3010 & 3171.0 & 108.6 \\
\hline AR (glucose only) & 8863 & 9565 & 9453 & 9006 & 9221.8 & 339.9 \\
\hline AR (supplemented) & 12135 & 12481 & 11991 & 11798 & 12101.3 & 288.4 \\
\hline AF (void) & 3197 & 3344 & 3003 & 2880 & 3106.0 & 205.4 \\
\hline AF (glucose only) & 10457 & 10846 & 10669 & 10838 & 10702.5 & 182.9 \\
\hline \multirow[t]{3}{*}{ AF (supplemented) } & 16211 & 11432 & 12949 & 9865 & 12614.3 & 2708.3 \\
\hline & \multicolumn{6}{|c|}{$120 \mathrm{~min}$} \\
\hline & Trial 1 & Trial 2 & Trial 3 & Trial 4 & AVG & STEV \\
\hline AR (void) & 1935 & 1954 & 1893 & 1845 & 1906.8 & 48.4 \\
\hline AR (glucose only) & 10951 & 10789 & 10962 & 10510 & 10803.0 & 210.7 \\
\hline AR (supplemented) & 10368 & 10213 & 10313 & 9998 & 10223.0 & 163.1 \\
\hline$A F$ (void) & 2254 & 2299 & 2344 & 2166 & 2265.8 & 76.0 \\
\hline AF (glucose only) & 9120 & 8964 & 8895 & 9065 & 9011.0 & 100.8 \\
\hline \multirow[t]{3}{*}{ AF (supplemented) } & 10485 & 10436 & 10520 & 10582 & 10505.8 & 61.4 \\
\hline & \multicolumn{6}{|c|}{$240 \min$} \\
\hline & Trial 1 & Trial 2 & Trial 3 & Trial 4 & AVG & STEV \\
\hline AR (void) & 668 & 721 & 712 & 645 & 686.5 & 36.1 \\
\hline AR (glucose only) & 11850 & 11742 & 10334 & 10417 & 11085.8 & 822.0 \\
\hline AR (supplemented) & 7301 & 7364 & 7650 & 7350 & 7416.3 & 158.2 \\
\hline AF (void) & 709 & 1287 & 861 & 1104 & 990.3 & 256.1 \\
\hline AF (glucose only) & 3843 & 4023 & 3797 & 3623 & 3821.5 & 164.4 \\
\hline AF (supplemented) & 3951 & 3642 & 3570 & 3378 & 3635.3 & 238.2 \\
\hline
\end{tabular}




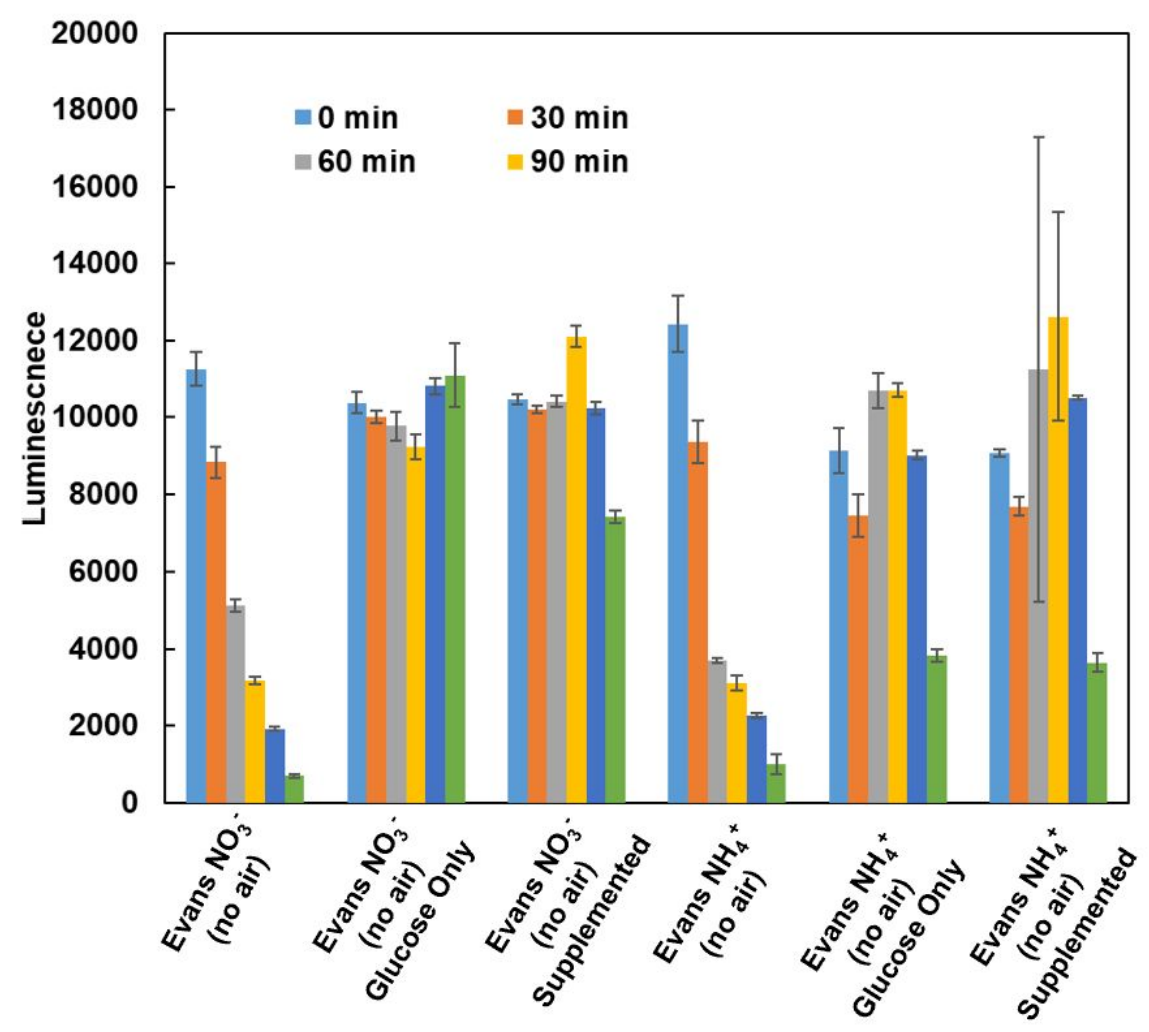

Supplement Figure 11 - raw luminescence experiment readings. AF (Evans with ammonium anaerobic) and AR (Evans with nitrate anaerobic) lysate CFPS reactions were compared. Reactions were stopped at various time points -0, 30, 60, 90 minutes. CFPS reactions were diluted 200 times. See Supplement Section 6 for details. "Supplemented" reactions contained both glucose and nitrite. "Glucose only" reactions were supplemented with glucose (40mM) alone. This is the third trial of this experiment (Supplement Table 16).

Third trial. In this trial, the experiment was setup as a replicate of the second trial, except that a third condition was added where supplementation was made only with glucose and not with nitrite. The "NA" (nitrate supplemented during growth) nomenclature corresponds with the AR lysate, while the "AM" (ammonium supplemented during cell growth) nomenclature corresponds with the AF lysate. The void condition consisted of the assay mix and CFPS reaction unsuplemented with either glucose or nitrite. The "Glu" condition consisted of the assay mix and CFPS reaction supplemented with glucose only. The "+" condition consisted of the assay mix and CFPS reactions supplemented with both glucose and nitrite.

To our surprise, the increase in ATP signal was attributed to the addition of glucose rather than nitrite. These results indicate that addition of glucose allows CFPS reactions to generate an abundance of ATP. In these three trials we did not see a statistically significant advantage to using AR lysate. The resolution of this method did not allow to determine whether the AR lysate generates more ATP than the AF lysate.

Additional investigations were not undertaken, since we had a limited amount of the AR and AF lysates prepared for this project, and did not have access to the fermentation equipment to generate new lysate easily. 


\section{Supplement 8 - Nitrate and Glucose gradient}

Additional control experiments were conducted where a gradient of glucose and a gradient of nitrate was added to CFPS reactions as a supplement. These experiments indicated that the addition of nitrate at higher concentrations decreases the performance of CFPS reactions, likely due to high osmolality. Addition of glucose does not provide a significant advantage across all concentrations. A slight uptick in performance (sfGFP yield) at $40 \mathrm{mM}$ glucose and $0 \mathrm{mM}$ nitrate was observed.

Supplement Table 17 - Reaction setup and sfGFP yield for the nitrate and glucose gradient reactions with the AF (Evans media supplemented with ammonium during anaerobic growth) lysate.

\begin{tabular}{|ccc|cccc|}
\hline & & & \multicolumn{4}{c|}{ RFU Yield } \\
RXN\# & Glucose (mM) & Nitrate (mM) & Trial 1 & Trial 2 & AVG & STDEV \\
\hline $\mathbf{1}$ & 0 & 0 & 15560 & 15404 & 15482 & 110.3 \\
$\mathbf{2}$ & 0.1 & 0 & 15421 & 15704 & 15562.5 & 200.1 \\
$\mathbf{3}$ & 1 & 0 & 15488 & 15256 & 15372 & 164.0 \\
$\mathbf{4}$ & 5 & 0 & 15363 & 15426 & 15394.5 & 44.5 \\
$\mathbf{5}$ & 40 & 0 & 17027 & 16881 & 16954 & 103.2 \\
$\mathbf{6}$ & 0 & 0.1 & 15113 & 14983 & 15048 & 91.9 \\
$\mathbf{7}$ & 0.1 & 0.1 & 14545 & 15121 & 14833 & 407.3 \\
$\mathbf{8}$ & 1 & 0.1 & 15022 & 15008 & 15015 & 9.9 \\
$\mathbf{9}$ & 5 & 0.1 & 15164 & 15103 & 15133.5 & 43.1 \\
$\mathbf{1 0}$ & 40 & 0.1 & 16990 & 15108 & 16049 & 1330.8 \\
$\mathbf{1 1}$ & 0 & 1 & 15700 & 15136 & 15418 & 398.8 \\
$\mathbf{1 2}$ & 0.1 & 1 & 14694 & 14505 & 14599.5 & 133.6 \\
$\mathbf{1 3}$ & 1 & 1 & 14767 & 15549 & 15158 & 553.0 \\
$\mathbf{1 4}$ & 5 & 1 & 14681 & 14611 & 14646 & 49.5 \\
$\mathbf{1 5}$ & 40 & 1 & 16560 & 16538 & 16549 & 15.6 \\
$\mathbf{1 6}$ & 0 & 5 & 15456 & 14555 & 15005.5 & 637.1 \\
$\mathbf{1 7}$ & 0.1 & 5 & 15196 & 14722 & 14959 & 335.2 \\
$\mathbf{1 8}$ & 1 & 5 & 14364 & 14306 & 14335 & 41.0 \\
$\mathbf{1 9}$ & 5 & 5 & 14141 & 14361 & 14251 & 155.6 \\
$\mathbf{2 0}$ & 40 & 5 & 15706 & 15761 & 15733.5 & 38.9 \\
$\mathbf{2 1}$ & 0 & 40 & 12370 & 11829 & 12099.5 & 382.5 \\
$\mathbf{2 2}$ & 0.1 & 40 & 10682 & 10516 & 10599 & 117.4 \\
$\mathbf{2 3}$ & 1 & 40 & 12023 & 11804 & 11913.5 & 154.9 \\
$\mathbf{2 4}$ & 5 & 40 & 11214 & 11178 & 11196 & 25.5 \\
$\mathbf{2 5}$ & 40 & 40 & 11711 & 11880 & 11795.5 & 119.5 \\
\hline
\end{tabular}


Supplement Table 18 - Reaction setup and SfGFP yield for the nitrate and glucose gradient reactions with the AR (Evans media supplemented with nitrate during anaerobic growth) lysate.

\begin{tabular}{|ccc|cccc|}
\hline & & & \multicolumn{4}{c|}{ RFU Yield } \\
RXN\# & Glucose (mM) & Nitrate (mM) & Trial 1 & Trial 2 & AVG & STDEV \\
\hline $\mathbf{1}$ & 0 & 0 & 9036 & 8700 & 8868 & 237.6 \\
$\mathbf{2}$ & 0.1 & 0 & 8104 & 8698 & 8401 & 420.0 \\
$\mathbf{3}$ & 1 & 0 & 8340 & 8233 & 8286.5 & 75.7 \\
$\mathbf{4}$ & 5 & 0 & 8247 & 8283 & 8265 & 25.5 \\
$\mathbf{5}$ & 40 & 0 & 9070 & 9065 & 9067.5 & 3.5 \\
$\mathbf{6}$ & 0 & 0.1 & 8164 & 8579 & 8371.5 & 293.4 \\
$\mathbf{7}$ & 0.1 & 0.1 & 9612 & 9252 & 9432 & 254.6 \\
$\mathbf{8}$ & 1 & 0.1 & 8630 & 8138 & 8384 & 347.9 \\
$\mathbf{9}$ & 5 & 0.1 & 8708 & 8383 & 8545.5 & 229.8 \\
$\mathbf{1 0}$ & 40 & 0.1 & 8251 & 8917 & 8584 & 470.9 \\
$\mathbf{1 1}$ & 0 & 1 & 9856 & 9355 & 9605.5 & 354.3 \\
$\mathbf{1 2}$ & 0.1 & 1 & 9209 & 8650 & 8929.5 & 395.3 \\
$\mathbf{1 3}$ & 1 & 1 & 8602 & 8672 & 8637 & 49.5 \\
$\mathbf{1 4}$ & 5 & 1 & 6529 & 6323 & 6426 & 145.7 \\
$\mathbf{1 5}$ & 40 & 1 & 8392 & 9014 & 8703 & 439.8 \\
$\mathbf{1 6}$ & 0 & 5 & 9599 & 9684 & 9641.5 & 60.1 \\
$\mathbf{1 7}$ & 0.1 & 5 & 9199 & 8954 & 9076.5 & 173.2 \\
$\mathbf{1 8}$ & 1 & 5 & 8758 & 8569 & 8663.5 & 133.6 \\
$\mathbf{1 9}$ & 5 & 5 & 8623 & 8879 & 8751 & 181.0 \\
$\mathbf{2 0}$ & 40 & 5 & 9586 & 9136 & 9361 & 318.2 \\
$\mathbf{2 1}$ & 0 & 40 & 6849 & 6503 & 6676 & 244.7 \\
$\mathbf{2 2}$ & 0.1 & 40 & 6604 & 6291 & 6447.5 & 221.3 \\
$\mathbf{2 3}$ & 1 & 40 & 6468 & 6381 & 6424.5 & 61.5 \\
$\mathbf{2 4}$ & 5 & 40 & 5433 & 5917 & 5675 & 342.2 \\
$\mathbf{2 5}$ & 40 & 40 & 5944 & 5694 & 5819 & 176.8 \\
\hline
\end{tabular}


Nitrate gradient (Glucose $0 \mathrm{mM}$ )

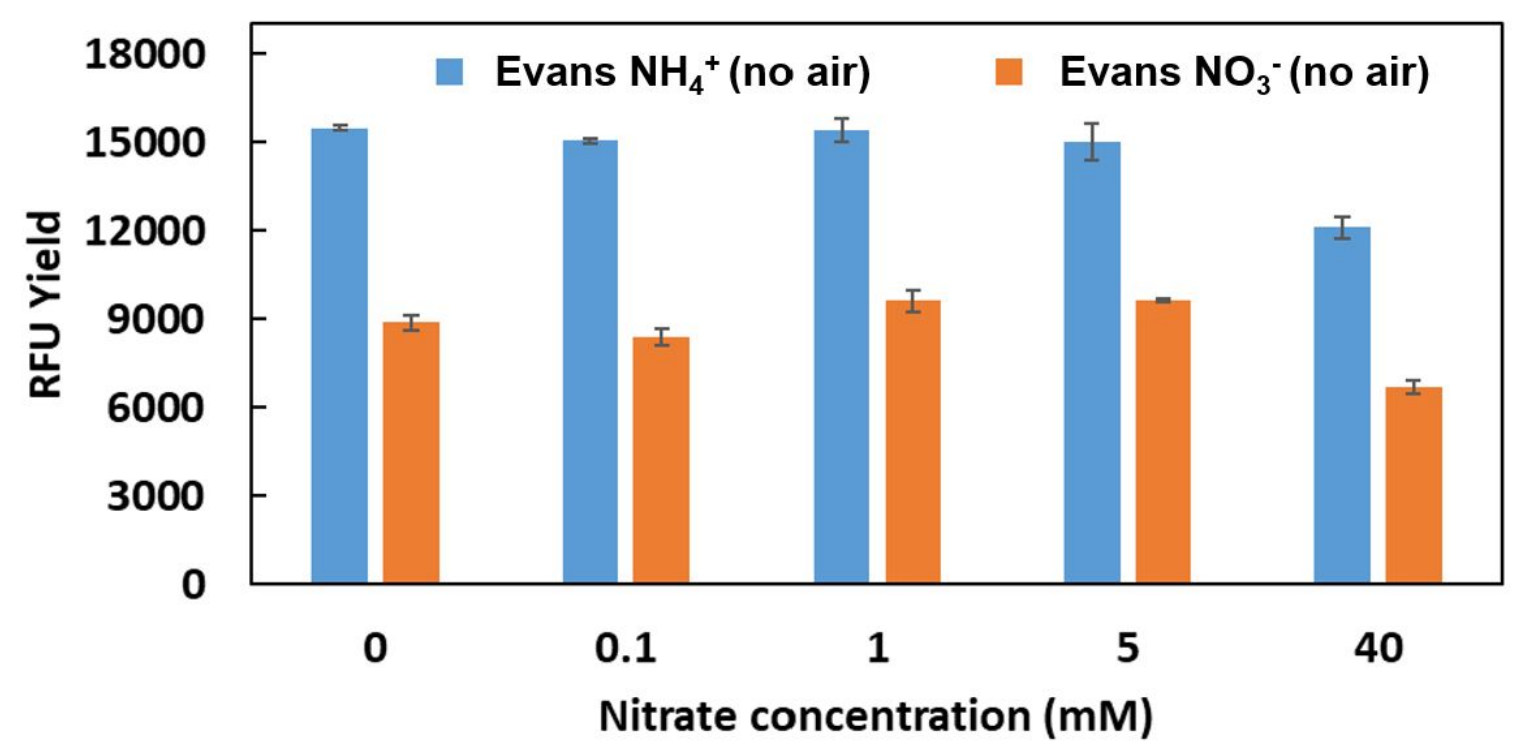

Supplement Figure 12 - sfGFP (RFU) yield of CFPS reactions with various nitrate concentrations supplemented (fixed glucose at OmM).

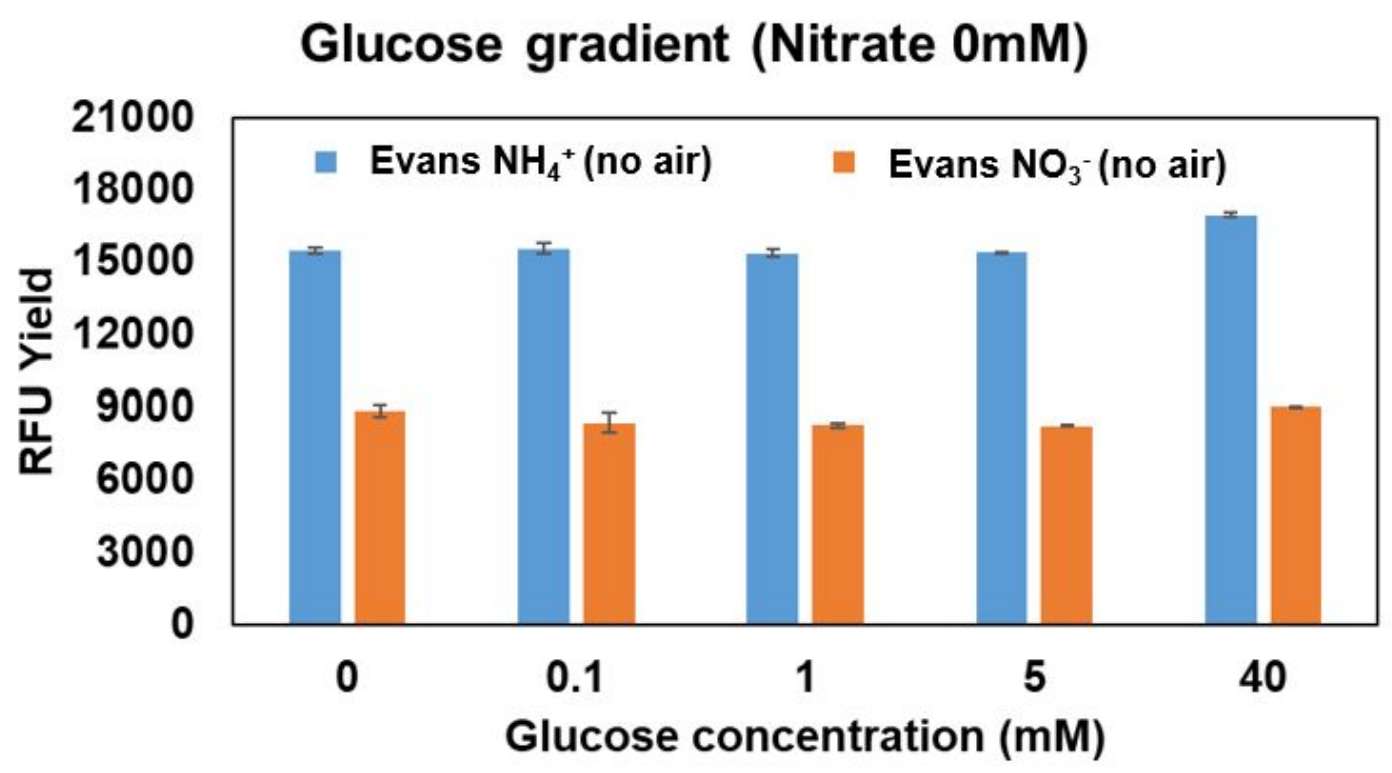

Supplement Figure 13 - sfGFP (RFU) yield of CFPS reactions with various glucose concentrations supplemented (fixed nitrate at OmM).

\section{Supplement 9 - Master Mix for CFPS reactions}

In these CFPS reactions we used the PANOXSP master mix composed according to the Supplement Table 19. 
Supplement Table 19 - The composition of the master mix.

\begin{tabular}{|l|r|}
\hline \multicolumn{1}{|c|}{ Reagent } & Final Concnetration \\
\hline $\mathrm{Mg}(\mathrm{GLU}) 2$ & $16 \mathrm{mM}$ \\
\hline $\mathrm{NH} 4(\mathrm{GLU})$ & $10 \mathrm{mM}$ \\
\hline $\mathrm{K}(\mathrm{GLU})$ & $175 \mathrm{mM}$ \\
\hline ATP & $1.2 \mathrm{mM}$ \\
\hline $\mathrm{GMP}$ & $0.85 \mathrm{mM}$ \\
\hline UMP & $0.85 \mathrm{mM}$ \\
\hline CMP & $0.85 \mathrm{mM}$ \\
\hline Folinic Acid & $0.034 \mathrm{mg} / \mathrm{mL}$ \\
\hline tRNA & $0.171 \mathrm{mg} / \mathrm{mL}$ \\
\hline NAD & $0.33 \mathrm{mM}$ \\
\hline CoA & $0.26 \mathrm{mM}$ \\
\hline K Oxalate & $2.7 \mathrm{mM}$ \\
\hline Putrescine & $1 \mathrm{mM}$ \\
\hline HEPES & $57 \mathrm{mM}$ \\
\hline Amino Acids & $2 \mathrm{mM}$ \\
\hline PEP Monopotassium & $33 \mathrm{mM}$ \\
\hline
\end{tabular}

\section{Supplement 10 - Comparing various lysates to commercial kits.}

In this experiment a standard 2xYTPG lysate was compared to minimal media aerobic (Evans $\mathrm{NH}^{+}$Air), AF capable (Evans $\mathrm{NH}^{+}$No air), AR capable (Evans NO3-) lysates. All of these lysates were also compared to kit reactions (same DNA concentration) from Promega (S30 T7 High-Yield Protein Expression System), and Arbor Biosciences (myTXTL).

The kit reactions were prepared according to the user manual. The same final concentration of DNA was used in CFPS reactions composed from the lysate prepared in our lab as well as in kit reactions. The reactions were performed twice. In the first trial CFPS reactions were incubated at $37^{\circ} \mathrm{C}$ for 2 hours (Supplement Table 20, and Figure 3). During this experiment we observed that myTXTL CFPS reactions took longer to begin expressing sfGFP, and protein expression did not stop after 2 hours of incubation. To investigate this further, we repeated this panel of CFPS reactions but conducted the experiment for 12 hours instead of 2 hours (Supplement Table 21, Supplement Figure 14). The key observation of these experiments was that at the 2 hours mark "Evans $\mathrm{NH}_{4}{ }^{+}-\mathrm{No}$ air" lysate outperforms all other reaction conditions, resulting in $595 \mu \mathrm{g} / \mathrm{ml}$ sfGFP yield (Supplement Table 20). This data was used for the Figure 3 of the main manuscript. However, in a separate experiment, after more prolonged incubation (12 hours instead of 2 hours), the myTXTL CFPS reaction eventually caught up to the "Evans $\mathrm{NH}_{4}{ }^{+}-\mathrm{No}$ air" condition (Supplement Figure 14), resulting in similar expression yield -558 and $560 \mu \mathrm{g} / \mathrm{ml}$ respectively (Supplement Table 21). 
Supplement Table 20 - The table of RFU yields of various CFPS lysates after incubation at 37C for 2 hours (Supplement 10).

\begin{tabular}{|c|c|c|c|c|c|c|c|}
\hline \multirow{2}{*}{ Lysate } & \multicolumn{5}{|c|}{ RFU Yield } & \multicolumn{2}{|c|}{ sfGFP ( $\mu \mathrm{g} / \mathrm{ml})$} \\
\hline & Trial 1 & Trial 2 & Trial 3 & AVG & STDEV & AVG & STDEV \\
\hline 2xYTPG & 3085 & 2827 & 2533 & 3048.0 & 113.1 & 274.0 & 24.9 \\
\hline $\begin{array}{c}\text { Evans } \mathrm{NH}_{4}^{+} \\
\text {(air) }\end{array}$ & 1853 & 1842 & 1510 & 1735.0 & 194.9 & 162.5 & 31.8 \\
\hline $\begin{array}{c}\text { Evans } \mathrm{NH}_{4}^{+} \\
\text {(no air) }\end{array}$ & 6929 & 6406 & 7171 & 6835.3 & 391.0 & 595.4 & 48.5 \\
\hline $\begin{array}{c}\text { Evans } \mathrm{NO}_{3}^{-} \\
\text {(no air) }\end{array}$ & & 2843 & 3456 & 3149.5 & 433.5 & 282.6 & 52.1 \\
\hline Promega & 4543 & 3627 & 3550 & 3906.7 & 552.4 & 346.8 & 62.2 \\
\hline myTXTL & 2527 & 3774 & 3562 & 3287.7 & 667.2 & 294.3 & 71.9 \\
\hline
\end{tabular}

Supplement Table 21 - The table of RFU yields of various CFPS lysates after incubation at 37 for 12 hours (Supplement 10).

\begin{tabular}{|c|c|c|c|c|c|c|c|}
\hline \multirow{2}{*}{ Lysate } & \multicolumn{5}{|c|}{ RFU Yield } & \multicolumn{2}{|c|}{ sfGFP ( $\mu \mathrm{g} / \mathrm{ml})$} \\
\hline & Trial 1 & Trial 2 & Trial 3 & AVG & STDEV & AVG & STDEV \\
\hline 2xYTPG & 4610 & 4372 & 5003 & 4661.67 & 318.66 & 410.91 & 42.33 \\
\hline $\begin{array}{c}\text { Evans } \mathrm{NH}_{4}^{+} \\
\text {(air) }\end{array}$ & 3289 & 3263 & 3302 & 3284.67 & 19.86 & 294.05 & 16.97 \\
\hline $\begin{array}{c}\text { Evans } \mathrm{NH}_{4}^{+} \\
\text {(no air) }\end{array}$ & 6320 & 6227 & 6705 & 6417.33 & 253.43 & 559.91 & 36.79 \\
\hline Promega & 3929 & 4574 & 3923 & 4142.00 & 374.14 & 366.81 & 47.04 \\
\hline myTXTL & 6489 & 6281 & 6406 & 6392.00 & 104.70 & 557.76 & 24.17 \\
\hline
\end{tabular}




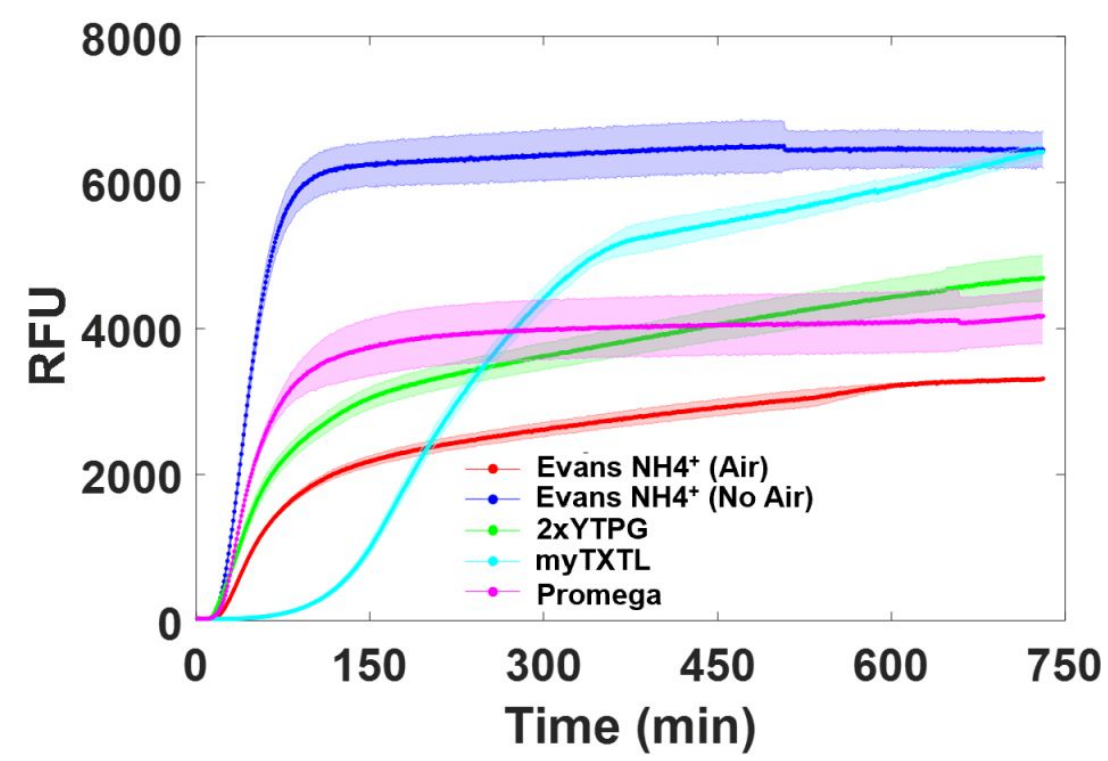

Supplement Figure 14 - sfGFP expression in various lysates (Supplement Table 21).

\section{Supplement 11 - Testing various reporter proteins.}

In order to further evaluate the productivity of the anaerobically prepared lysate (AF capable lysate), and a standard 2xYTPG lysate, CFPS reactions were formulated to test expression of 3 additional reporter proteins - mCherry, Subtilisin BPN and Chitinase A.

mCherry. The fluorescence of mCherry was acquired with excitation/emission $587 \mathrm{~nm} / 610 \mathrm{~nm}$, bandwidth of $10 \mathrm{~nm}$ and gain set at 60 (BioTek Synergy neo2). The CFPS reactions contained either the $2 x Y T P G$ or the AF (Evans NH4+ No air) lysates in otherwise identical conditions. These reactions were incubated at $37 \mathrm{C}$ for 12 hours and the fluorescence was measured every 5 minutes. Protein expression yield is reported as the difference in fluorescence at the start of the reaction and after 12 hours (Supplement Table 22). A PCR amplified linear DNA template was used in this experiment, and the DNA sequence is listed below.

Supplement Table 22 - The table of RFU yields of various CFPS lysates after incubation at 37 for 12 hours (Supplement 11).

\begin{tabular}{|c|ccccc|}
\hline \multirow{2}{*}{ Lysate } & \multicolumn{5}{|c|}{ RFU Yield } \\
& Trial 1 & Trial 2 & Trial 3 & AVG & STDEV \\
\hline 2xYTPG & 86 & 90 & 94 & 90.0 & 4.0 \\
Evans $\mathrm{NH}_{4}^{+}$(no air) & 262 & 254 & 263 & 259.7 & 4.9 \\
\hline
\end{tabular}

mCherry - 993bp 
GTGACACAGGATTCTAGCCTGCAAGACGGTGAGTTCATCTATAAAGTGAAACTGCGTGGCACGAATTTTCCGAGT GATGGCCCGGTTATGCAGAAAAAAACGATGGGTTGGGAGGCCTCTAGTGAGCGTATGTATCCAGAAGATGGCGC TCTGAAAGGCGAAATCAAACAGCGTCTGAAACTGAAAGATGGTGGCCACTATGATGCCGAAGTGAAAACCACGTA TAAAGCCAAAAAACCTGTCCAACTGCCTGGTGCCTATAACGTTAACATCAAACTGGACATCACCTCACACAATGAG GACTATACGATCGTGGAGCAGTATGAGCGTGCTGAAGGACGTCATTCTACCGGTGGTATGGATGAGCTGTATAAA GTCGACCGGCTGCTAACAAAGCCCGAAAGGAAGCTGAGTTGGCTGCTGCCACCGCTGAGCAATAACTAG CATAACCCCTTGGGGCCTCTAAACGGGTCTTGAGGGGTTTTTTGCTGAAAGCGAGACTAAGCTTTAAACTTCGG

\section{T7 Promoter}

\section{RBS}

Start

Protein Sequence - mCherry and mVenus from Roberta Lentini et al. ACS Synth Bio paper

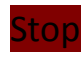

\section{T7 Terminator}

Circularization site - HindIII Digest

Subtilisin BPN. The Subtilisin expression was performed with CFPS reactions formulated identically to that of the mCherry expression experiment with the exception that the DNA template contained the protein sequence of Subtilisin BPN. CFPS reactions were incubated at $30 \mathrm{C}$ for 18 hours. At the end of the incubation period CFPS reactions were transferred to microcentrifuge tubes and stored on ice to minimize non-productive auto degradation of Subtilisin. Due to high yield of Subtilisin expression, the CFPS reactions were diluted 10X with S30 buffer. The subtilisin assay (100ul volume) consisted of 5ul diluted CFPS reaction, $2 u$ l of the $10 \mathrm{mM}$ suc-AAPF-pNA substrate and $93 \mathrm{ul}$ of the S30 buffer. The assay was conducted in triplicates. Assay mixtures were incubated at $37 \mathrm{C}$ for 15 minutes and the increase in absorbance at 410nm (hydrolysis of the AAPF peptide and consecutive release of pNA) was measured. The initial rate of the increase in absorbance at $410 \mathrm{~nm}$ was measured to determine the activity of Subtilisin expressed in each CFPS reaction (Supplement Table 23). Since the experimental condition of CFPS Subtilisin expression were identical with the exception for the nature of the lysate (aerobic 2xYTPG vs anaerobic AF lysate) - it was assumed that the proteolytic activity of Subtilisin correlated directly with the expression yield. A CFPS reaction without DNA was used as a negative control to measure the background activity of endogenous proteases. A PCR amplified linear DNA template was used in this experiment, and the DNA sequence is listed below. 
Supplement Table 23 - Subtilisin activity (relative absorbance units at 410nm per minute) of various CFPS lysates (Supplement 11).

\begin{tabular}{|c|ccccc|}
\hline \multirow{2}{*}{ Lysate } & \multicolumn{5}{|c|}{ Subtilisin Activity (RAU410/second) } \\
& Trial 1 & Trial 2 & Trial 3 & AVG & STDEV \\
\hline 2xYTPG & 0.000394 & 0.000385 & 0.000412 & 0.000397 & $1.38 \mathrm{E}-05$ \\
Evans $\mathrm{NH}_{4}{ }^{+}$(no air) & 0.001018 & 0.000994 & 0.001061 & 0.001025 & $3.38 \mathrm{E}-05$ \\
Negative Control & 0 & $5.56 \mathrm{E}-06$ & $-5.6 \mathrm{E}-06$ & 0 & $5.56 \mathrm{E}-06$ \\
\hline
\end{tabular}

Subtilisin -1344 bp

AGCGCTATTAAAGCTTCGAAATTAATACGACTCACTATAGGGAGACCACAACGGTTTCC CTCTAGAAATAATTTTGTTTAACTTTAAGAAGGAGATATACATATGGCAGGTAAAAGTAACGGCGAGAAAAAATA CATCGTTGGCTTCAAACAAACGATGTCGACCATGAGCGCAGCGAAAAAGAAAGATGTCATCAGCGAAAAAGGCG GTAAAGTGCAGAAACAATTCAAATACGTTGACGCGGCCAGTGCCACCCTGAATGAAAAAGCAGTGAAAGAACTG AAGAAAGATCCGTCCGTGGCGTACGTTGAAGAAGACCATGTTGCTCACGCGTATGCCCAGTCCGTTCCGTACGGT GTCTCACAAATTAAAGCACCGGCTCTGCATTCGCAGGGCTATACCGGTAGCAACGTTAAAGTCGCGGTGATTGATA GCGGCATCGACAGTTCCCACCCGGATCTGAAAGTTGCGGGCGGTGCCAGCATGGTGCCGAGCGAAACCAATCCGT TCCAGGACAACAATAGCCATGGCACGCATGTGGCGGGTACCGTTGCAGCTCTGAACAATTCTATTGGCGTCCTGG GTGTGGCACCGTCTGCTAGTCTGTATGCGGTTAAAGTCCTGGGCGCCGATGGCTCTGGCCAGTACAGTTGGATTAT CAACGGTATTGAATGGGCGATCGCCAACAATATGGATGTGATCAATATGAGCCTGGGCGGTCCGTCCGGTTCAGC CGCACTGAAAGCAGCTGTCGACAAAGCAGTTGCTTCCGGTGTGGTTGTTGTGGCCGCAGCCGGTAACGAAGGCAC GTCAGGCTCATCGAGCACCGTGGGTTATCCGGGCAAATACCCGTCGGTTATTGCGGTCGGTGCCGTGGATTCTAG TAATCAGCGTGCGAGCTTTTCCTCAGTTGGCCCGGAACTGGACGTTATGGCCCCGGGTGTCTCTATTCAAAGTACG CTGCCGGGTAACAAATATGGCGCGTACAATGGTACCAGCATGGCATCACCGCATGTGGCTGGTGCTGCGGCCCTG ATCCTGAGCAAACACCCGAACTGGACGAATACCCAGGTTCGCTCGAGCCTGGAAAACACCACGACCAAACTGGGC GATTCTTTCTATTACGGCAAAGGTCTGATCAATGTTCAGGCAGCTGCGCAATAATAA GTCGACCGGCTGCTAACAA AGCCCGAAAGGAAGCTGAGTTGGCTGCTGCCACCGCTGAGCAATAACTAGCATAACCCCTTGGGGCCTCTAAACG GGTCTTGAGGGGTTTTTTGCTGAAAGCGAGACTAAGCTTTAAACTTCGG

Needed Genetic Elements by color code:

T7 Promoter

RBS

Start

Protein Sequence

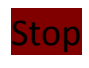

T7 Terminator

Circularization site - HindIII Digest 
Chitinase A. The Chitinase expression was performed with CFPS reactions formulated identically to that of the mCherry and Subtilisin expression experiments with the exception that the DNA template contained the protein sequence of Subtilisin BPN. CFPS reactions were incubated at $30 \mathrm{C}$ for 18 hours. The Chitinase assay (100ul volume) consisted of 10ul of the MUF-triNAG (1mM) substrate (Cayman Chemical CAS: 53643-13-3), 5ul of CFPS lysate and 85ul of the S30 buffer. In that assay, Chitinase would hydrolyze the substrate which results in the increase in fluorescence. The rate of the increase in fluorescence (excitation/emission 360nm/445nm, bandwidth 20 and gain 100) over 15 minutes at 37C was used to measure activity of Chitinase expressed in different CFPS reactions (Supplement Table 24). Since the experimental condition of CFPS Chitinase expression were identical with the exception for the nature of the lysate (aerobic 2xYTPG vs anaerobic AF lysate) - it was assumed that the hydrolytic activity of Chitinase correlated directly with the expression yield. The experiment was conducted in triplicates.

A PCR amplified linear DNA template was used in this experiment, and the DNA sequence is listed below.

Supplement Table 23 - Chitinase A activity (RFU per second) of various CFPS lysates (Supplement 11).

\begin{tabular}{|c|ccccc|}
\hline \multirow{2}{*}{ Lysate } & \multicolumn{5}{|c|}{ Activity (RFU/seconds) } \\
& Trial 1 & Trial 2 & Trial 3 & AVG & STDEV \\
\hline 2xYTPG & 0.418 & 0.391 & 0.372 & 0.394 & 0.023 \\
Evans $\mathrm{NH}_{4}{ }^{+}$(no air) & 1.260 & 1.111 & 1.250 & 1.207 & 0.083 \\
Negative Control & -0.137 & -0.113 & -0.129 & -0.126 & 0.012 \\
\hline
\end{tabular}

Chitinase (prchia A) - 1101 base pairs

AGCGCTATTAAAGCTTCGAAATTAATACGACTCACTATAGGGAGACCACAACGGTTTCC CTCTAGAAATAATTTTGTTTAACTTTAAGAAGGAGATATACATATGAAGGTGTTCAGAGAGTATATTGGTGCTTTA TATAATGGCGTACAATTTACAGACGTACCAATCAACTCCGGTGTGACATTCCATTTTATTCTGGCCTTTGCTATTGAC TATACGAGCGCGGCAGCGGCGACAAACGGAGTCTTCAATATTTACTGGCAGAACTCTGTTCTTACACCTGCGGCTG TCCAAGCGATTAAGGCACAGCACTCGAATGTCAAGGTGATGGTTTCGTTAGGAGGGGACACCATTAGCGGCTCGC CTGTCCAATTCACGGCGACAAGTGTCTCATCCTGGGTTGCGAATGCAGTATCATCCCTGACTTCGTTGATTAATCAG TACCACTTGGACGGCATAGATATTGACTACGAGCACTTCGACCAGGTAAGTACATCTACCTTTGTTTCTTGCATTGG GCAGTTAATTACACAATTAAAGGCGAACAACGTAATATCGGTTGCGTCGATAGCTCCTTTTGATGGTGTAGAGTCA CAATACACCGCACTTTTTTGGTCAATACTCTTCCGTTATCGACTTAGTTAATTTCCAGTTTTACTCATATGGCGCCGGT ACAAGCGCTTCACAGTACGTGTCGCTGTATAATACTGCCGCGAGTAAATATGGAGGGGGAGCGAAGGTACTGGC AAGTTTTAGTACGGGTGGAGTCGGACCAGCTCCGTCCACCGTTCTGTCCGCGTGCCAGCAACTTAAATCGAGTGGC ACCCTTCCAGGAATCTTTATTTTCTCAGCTGATGGGAGCTACGCGAGTAGCGCGAAGTTTCAGTATGAGCAACAAG CACAGACGTTATTAACCTCATAATAA GTCGACCGGCTGCTAACAAAGCCCGAAAGGAAGCTGAGTTGGCTGCTGC CACCGCTGAGCAATAACTAGCATAACCCCTTGGGGCCTCTAAACGGGTCTTGAGGGGTTTTTTGCTGAAAGCGAG ACTAAGCTTTAAACTTCGG 